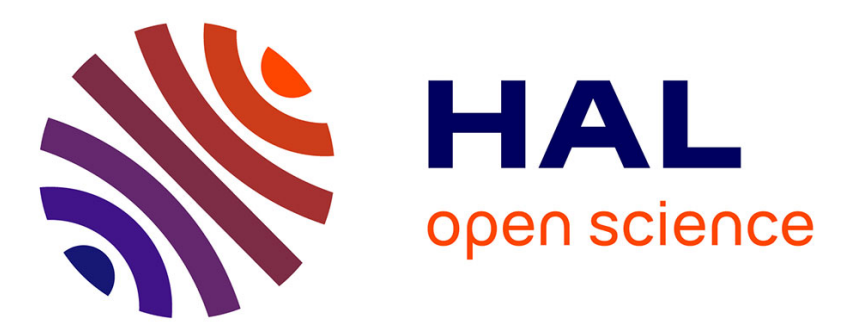

\title{
Scalar Auxiliary Variable/Lagrange multiplier based pseudospectral schemes for the dynamics of nonlinear Schrödinger/Gross-Pitaevskii equations
}

Xavier Antoine, Jie Shen, Qinglin Tang

\section{- To cite this version:}

Xavier Antoine, Jie Shen, Qinglin Tang. Scalar Auxiliary Variable/Lagrange multiplier based pseudospectral schemes for the dynamics of nonlinear Schrödinger/Gross-Pitaevskii equations. Journal of Computational Physics, 2021, 437, pp.110328. 10.1016/j.jcp.2021.110328 . hal-02940080

\author{
HAL Id: hal-02940080 \\ https://hal.science/hal-02940080
}

Submitted on 16 Sep 2020

HAL is a multi-disciplinary open access archive for the deposit and dissemination of scientific research documents, whether they are published or not. The documents may come from teaching and research institutions in France or abroad, or from public or private research centers.
L'archive ouverte pluridisciplinaire HAL, est destinée au dépôt et à la diffusion de documents scientifiques de niveau recherche, publiés ou non, émanant des établissements d'enseignement et de recherche français ou étrangers, des laboratoires publics ou privés. 


\title{
Scalar Auxiliary Variable/Lagrange multiplier based pseudospectral schemes for the dynamics of nonlinear Schrödinger/Gross-Pitaevskii equations
}

\author{
Xavier Antoine ${ }^{\mathrm{a}}$, Jie Shen ${ }^{\mathrm{b}}$, Qinglin Tang ${ }^{\mathrm{c}}$ \\ ${ }^{a}$ Université de Lorraine, CNRS, Inria, IECL, F-54000 Nancy, France. \\ ${ }^{b}$ Department of Mathematics, Purdue University, West Lafayette, IN 47907, USA \\ ${ }^{c}$ School of Mathematics, State Key Laboratory of Hydraulics and Mountain River Engineering, Sichuan \\ University, Chengdu 610064, China
}

\begin{abstract}
In this paper, based on the Scalar Auxiliary Variable (SAV) approach [40, 41] and a newly proposed Lagrange multiplier (LagM) approach [21, 20] originally constructed for gradient flows, we propose two linear implicit pseudo-spectral schemes for simulating the dynamics of general nonlinear Schrödinger/Gross-Pitaevskii equations. Both schemes are of spectral/second-order accuracy in spatial/temporal direction. The SAV based scheme preserves a modified total energy and approximate the mass to third order (with respect to time steps), while the LagM based scheme could preserve exactly the mass and original total energy. A nonlinear algebraic system has to be solved at every time step for the LagM based scheme, hence the SAV scheme is usually more efficient than the LagM one. On the other hand, the LagM scheme may outperform the SAV ones in the sense that it conserves the original total energy and mass and usually admits smaller errors. Ample numerical results are presented to show the effectiveness, accuracy and performance of the proposed schemes.

Keywords: real-time dynamics; nonlinear Schrödinger equation; Gross-Pitaevskii equation; explicit time scheme; Scalar Auxiliary Variables; Lagrange multiplier; pseudospectral method
\end{abstract}

\section{Contents}

\begin{tabular}{llr}
\hline 1 & Introduction & 2
\end{tabular}

\begin{tabular}{|lll}
2 & Two efficient SAV-based pseudospectral discretization schemes & 4
\end{tabular}

2.1 Original Scalar Auxiliary Variable (SAV) approach . . . . . . . . . . . . . 5

2.2 SAV via Lagrange multiplier approach . . . . . . . . . . . . . . . . . . 8

2.3 Two typical choices of energy splitting . . . . . . . . . . . . . . . . . . . . . 10

2.4 Pseudospectral discretization $\ldots \ldots \ldots \ldots \ldots \ldots \ldots$

Email addresses: xavier.antoine@univ-lorraine.fr (Xavier Antoine), shen7@purdue.edu (Jie Shen), qinglin_tang@163.com (Qinglin Tang) 


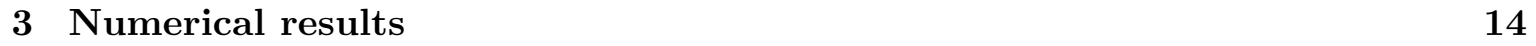

3.1 One-dimensional case . . . . . . . . . . . . . . . . . . . . . . . . . . . 15

3.2 Two-dimensional case $\ldots \ldots \ldots \ldots \ldots$

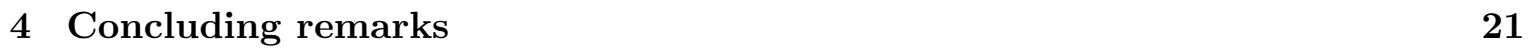

\section{Introduction}

Solving the $d$-dimensional $(d=2$ or 3 ) dimensionless GPE [1, 5, 7, 28, 33, 34 for BoseEinstein Condensation (BEC), with a rotational term [5, 7, 13, 15, 27, 34] and High-Order Interaction (HOI) [23, 25, 36] leads to the following initial boundary-value problem: for a given initial state $\psi_{0}$, find the complex-valued wave function $\psi(\mathbf{x}, t)$ solution to

$$
\left\{\begin{array}{l}
i \partial_{t} \psi(\mathbf{x}, t)=\left[-\frac{1}{2} \nabla^{2}+V(\mathbf{x})-\omega L_{z}+f\left(|\psi|^{2}\right)-\lambda \nabla^{2}|\psi|^{2}\right] \psi(\mathbf{x}, t), \quad \mathbf{x} \in \mathbb{R}^{d}, t \geq 0, \\
\psi(\mathbf{x}, t=0)=\psi_{0}(\mathbf{x}), \quad t \geq 0,
\end{array}\right.
$$

where $\mathbf{x}:=(x, y, z) \in \mathbb{R}^{d}(:=(x, y)$ in $2 \mathrm{D})$ and $t>0$ are the space and time variables, respectively. Denoting by $\nabla$ the gradient operator, $\nabla^{2}$ is then the laplacian and $V(\mathbf{x})$ is a function corresponding to the potential. The real-valued function $f(\rho)=F^{\prime}(\rho)$ models the nonlinearity. For example, a standard situation arises with the cubic case which reads as

$$
F(\rho)=\beta \rho^{2} / 2,
$$

where $\beta$ is the nonlinearity strength which is positive for a repulsive interaction and negative for an attractive interaction. If $\rho$ is the density function such that $\rho=|\psi|^{2}, f$ is then simply defined by $f(\rho)=\beta \rho$. When a HOI is included into (1.1) [23, 25, 36], the constant $\lambda$ is called the HOI strength and $\beta$ is the contact interaction strength. In addition, the realvalued constant $\omega$ represents the rotating frequency. The operator $L_{z}=i\left(y \partial_{x}-x \partial_{y}\right)$ is then the $z$-component of the angular momentum [5, 7]. When $\omega=\lambda=0$ and $f$ is cubic, (1.1) simplifies to

$$
i \partial_{t} \psi(\mathbf{x}, t)=\left[-\frac{1}{2} \nabla^{2}+V(\mathbf{x})+\beta|\psi|^{2}\right] \psi(\mathbf{x}, t), \quad \mathbf{x} \in \mathbb{R}^{d}, \quad t \geq 0,
$$

and is called the NonLinear cubic Schrödinger Equation (NLSE). Let us remark that many more complex models involving GPEs are widely used in the literature [5, 7], including in particular nonlocal dipolar interactions or multi-components gases.

For the GPE (1.1), the following two important invariants hold. First, we have the mass conservation

$$
\mathcal{N}(t)=\mathcal{N}(\psi(\cdot, t))=: \int_{\mathbb{R}^{d}}|\psi(\mathbf{x}, t)|^{2} d \mathbf{x} \equiv \int_{\mathbb{R}^{d}}\left|\psi_{0}(\mathbf{x})\right|^{2} d \mathbf{x}=\mathcal{N}(t=0) .
$$

Let us define now the total energy $\mathcal{E}_{\text {tot }}$ as the sum of the kinetic, potential, rotation and interaction energies

$$
\begin{aligned}
\mathcal{E}_{\text {tot }}(\cdot, t) & =\mathcal{E}_{\text {tot }}(\psi(t))=\int_{\mathbb{R}^{d}}\left[\frac{1}{2}|\nabla \psi|^{2}+V(\mathbf{x})|\psi|^{2}-\omega \operatorname{Re}\left(\bar{\psi} L_{z} \psi\right)+F(\rho)+\frac{\lambda}{2}|\nabla \rho|^{2}\right] d \mathbf{x} \\
& =\mathcal{E}_{\text {kin }}(t)+\mathcal{E}_{\text {pot }}^{V}(t)+\mathcal{E}_{\text {rot }}(t)+\mathcal{E}_{\text {int }}(t),
\end{aligned}
$$


for $t>0$. Then, 1.1 can be written as

$$
i \partial_{t} \psi(\mathbf{x}, t)=\frac{\delta \mathcal{E}_{\mathrm{tot}}}{\delta \bar{\psi}}
$$

which implies that $\mathcal{E}_{\text {tot }}$ is conserved, i.e.

$$
\mathcal{E}_{\text {tot }}(t)=\mathcal{E}_{\text {tot }}(0), \quad \forall t>0
$$

In this paper, we denote by $\bar{a}$ the complex conjugate of a complex-valued number $a$, and by $\operatorname{Re}(a)$ and $\operatorname{Im}(a)$ its respective real and imaginary parts.

Various schemes have been proposed to simulate the NLSE/GPE. For the sake of conciseness, we however only cite some of these references without being exhaustive. Concerning the spatial discretization, several possibilities exist (see e.g. [2, 5, 7]). Here, we focus on pseudospectral approximation schemes which are known to be well-adapted to solving NLSE/GPE with high-accuracy [2, 3, 4, 5, 7, 9, 11, 12, 13, 15, 19, 43, 44, 45]. Concerning the time discretization, different directions can be investigated [2, 5, 7], each method getting is pros and cons, like e.g. implicit/explicit, nonlinear/linear, unconditionally or conditionally stable, order of accuracy, computational cost. In addition, some of them conserve both the mass and the energy (together with its discrete version), and possibly other dynamical properties (see e.g. [2]). For example, the Crank-Nicolson (CN) scheme [2, 24], which is fully nonlinear and then implicit, leads to high computational costs when using a fixed-point method to resolve the nonlinearity. If the nonlinear term in the $\mathrm{CN}$-scheme is made explicit, then one gets the semi-implicit scheme [2, 7, 8, also called leap-frog scheme. While the CN scheme is mass and energy conserving (up to the a priori error on the fixed-point method), the semi-implicit scheme does not preserve the mass nor the energy. In [16], a second-order unconditionally stable relaxation scheme was introduced for the nonlinear cubic Schrödinger equation. It was first proved that the scheme preserves the mass and a modified energy for the cubic case, and it was recently extended to other power law nonlinearities [17]. In addition, the scheme is semi-implicit and can efficiently solve a large class of GPEs by coupling the pseudo-spectral scheme [5] with a preconditioned Krylov iterative subspace solver (usually GMRES [37, 38]). In [3], high-order IMEX (IMplicit-EXplicit) pseudo-spectral schemes with adaptive time stepping were presented for various kinds of systems of GPE equations, even with rotation terms. The schemes appear to be explicit and efficient, and potentially interesting when compared with splitting-schemes. Nevertheless, they do not conserve exactly the mass and the energy. In [31], the authors recently introduced a mass and energy conservative high-order diagonally implicit Runge-Kutta scheme for the cubic 1D and 2D nonlinear Schrödinger equations. However, it is not clear how to extend this scheme to the very general situation of interest in our paper, most particularly concerning the nonlinearity. Alternatively, time-splitting schemes can be used to solve NLSE/GPEs in some situations [5, 17, 11, 13, 19, 43]. In the case where the potential and nonlinearity can be integrated exactly, then the scheme is explicit and efficient, and unconditionally stable. When the rotational term is included into the equation, ADI methods [7] or techniques based on the Lagrangian change of coordinates to rewrite the equation without the rotation operator but with a time-dependent potential [12] can be developed. High-order splitting schemes can also be built [5, 7, 19, 32, 43, 46]. Concerning the second-order scheme, it is known to exactly 
preserve the mass but not the energy, which is accurately conserved when using FFT-based pseudo-spectral schemes [2, 7].

In all the above examples, it is clear that designing a time discretization directly affects the questions of accuracy, stability, efficiency and the existence of exact conservation laws. Therefore, there is always a trade-off when considering the time discretization and most specifically its nonlinear/linear implicit or explicit property, in particular in the pseudospectral framework. The unconditionally stable second-order time-splitting scheme is fully explicit, the mass conservation holds but the energy conservation property is lost. Recently, a powerful approach called the scalar auxiliary variable (SAV) was proposed for dissipative gradient flows [39, 40, 41], and was quickly successfully developed for other systems [22, 30]. Very recently, the SAV approach was also extended to the conservative system [18, 26, 42] and a SAV via Lagrange multiplier approach [20, 21] is constructed for gradient flows to deal with constraints.

The aim of this paper is to analyze the SAV approach for solving the NLSE/GPE (1.1). To this end, we derive two schemes, one based on the original SAV method and a second one that involves Lagrange multipliers for the conservation laws. Both schemes are secondorder in time and linearly implicit. In addition, we show that when combined with the highly accurate pseudospectral approximation in space, both schemes finally lead to fully explicit diagonal systems when using a well-suited energy splitting. The two schemes are then numerically validated on various $1 \mathrm{D}$ and $2 \mathrm{D}$ problems to illustrate their properties.

The structure of the paper is the following. In subection 2.1, we introduce a first SAV scheme and its linearly implicit semi-discretization in time. We also analyze in details the conservation properties, showing that the scheme conserves a modified energy and approximately preserves the mass at order $(\Delta t)^{3}$. We also propose in subsection 2.2 another linearly implicit semi-discrete SAV scheme with Lagrange multipliers to enforce the mass and energy conservation laws. We discuss in subsection 2.3 the way the energy splitting can be chosen in the SAV approach. In subsection 2.4, the pseudospectral approximation in space of the SAV schemes is developed showing that they can both made fully explicit. Numerical examples are reported in Section 3, first in one-dimension for several kinds of nonlinearities, and then in 2D for the GPE with rotation term. Finally, we conclude in Section 4.

\section{Two efficient SAV-based pseudospectral discretization schemes}

Here, we propose two semi-discrete schemes based on a orignal SAV approach and a SAV formulation with Lagrange multipliers to force the conservation constraints in subsections 2.1 and 2.2 , respectively. To this end, we start with a generic splitting of the free energy as

$$
\mathcal{E}_{\text {tot }}(\psi):=\mathcal{E}_{0}(\psi)+\mathcal{E}_{1}(\psi)
$$

where $\mathcal{E}_{0}(\phi)$ consists of usually quadratic terms such that

$$
\mathcal{L} \psi:=\frac{\delta \mathcal{E}_{0}(\psi)}{\delta \bar{\psi}}
$$

with $\mathcal{L}$ being a self-adjoint linear non-negative operator, and $\mathcal{E}_{1}(\psi)$ is bounded from below, i.e. there exists a strictly positive constant $\mathcal{E}_{c}>0$ such that $\mathcal{E}_{1}(\psi)+\mathcal{E}_{c}>0$. We will 
specify two possible splittings in subsection 2.3 , which one to use will depend on the choice of the spatial discretization and possibly the rotating speed $\omega$. We set $\psi^{n}=: \psi\left(\cdot, t_{n}\right)$ at discrete time $t_{n}=n \Delta t$, for the constant time step $\Delta t$. The inner product between two square-integrable functions $f$ and $g$ is

$$
\langle f, g\rangle=: \int_{\mathbb{R}^{d}} f \bar{g} d \mathbf{x} .
$$

\subsection{Original Scalar Auxiliary Variable (SAV) approach}

We first construct a linear scheme based on the original SAV approach [39, 40] for (1.1). We introduce the SAV function $\gamma(t)$ and the function $g(\psi)$ defined by

$$
\gamma(t)=: \sqrt{\mathcal{E}_{1}(\psi)+\mathcal{E}_{c}}, \quad g(\psi):=\frac{1}{\sqrt{\mathcal{E}_{1}(\psi)+\mathcal{E}_{c}}} \frac{\delta \mathcal{E}_{1}(\psi)}{\delta \bar{\psi}} .
$$

The original GPE (1.1) could be equivalently rewritten as

$$
\left\{\begin{array}{l}
i \partial_{t} \psi(\mathbf{x}, t)=\mathcal{L} \psi+g(\psi) \gamma(t), \quad \mathbf{x} \in \mathbb{R}^{d}, \quad t \geq 0, \\
\frac{d}{d t} \gamma(t)=\frac{1}{2 \sqrt{\mathcal{E}_{1}(\psi)+\mathcal{E}_{c}}} \frac{d}{d t} \mathcal{E}_{1}(\psi)=\operatorname{Re}\left\langle g(\psi), \partial_{t} \psi\right\rangle, \quad t \geq 0
\end{array}\right.
$$

for $\psi_{0}$ given and $\gamma_{0}=\gamma(t=0):=\sqrt{\mathcal{E}_{1}\left(\psi_{0}(\cdot, t)\right)+\mathcal{E}_{c}}$. Taking the inner product of the first equation with $\partial_{t} \psi$, then considering the real parts of the resulting equation and using the second equation of 2.11, we obtain the conservation of the modified energy

$$
\frac{d}{d t} \mathcal{E}_{\text {mod }}(t)=0, \quad \text { with } \quad \mathcal{E}_{\text {mod }}(t)=: \mathcal{E}_{0}(t)+|\gamma(t)|^{2} .
$$

From the definition of $\gamma(t)$ in 2.10 , the modified total energy is such that

$$
\mathcal{E}_{\text {mod }}(t)=\mathcal{E}_{0}(t)+|\gamma(t)|^{2}=\mathcal{E}_{\text {tot }}(t)+\mathcal{E}_{c}
$$

i.e. $\mathcal{E}_{\text {mod }}$ is equal to the original total energy $\mathcal{E}_{\text {tot }}$ up to the constant $\mathcal{E}_{c}$.

Standard semi-implicit scheme could then be applied to discretize the SAV system (2.11). We propose to use a second-order Crank-Nicolson Adam-Bashforth (CN-AB) scheme that reads as

$$
\begin{cases}\frac{i}{\Delta t}\left(\psi^{n+1}-\psi^{n}\right)=\mathcal{L} \psi^{n+\frac{1}{2}}+\tilde{g}^{n+\frac{1}{2}} \gamma^{n+\frac{1}{2}}, & \mathbf{x} \in \mathbb{R}^{d}, \\ \gamma^{n+1}-\gamma^{n}=\operatorname{Re}\left\langle\tilde{g}^{n+\frac{1}{2}}, \psi^{n+1}-\psi^{n}\right\rangle, & n \geq 1\end{cases}
$$

with $\psi^{0}=\psi_{0}$ and

$$
\tilde{\psi}^{n+\frac{1}{2}}=\frac{3}{2} \psi^{n}-\frac{1}{2} \psi^{n-1}, \quad \tilde{g}^{n+\frac{1}{2}}=g\left(\tilde{\psi}^{n+\frac{1}{2}}\right), \quad \nu^{n+\frac{1}{2}}=\frac{\nu^{n+1}+\nu^{n}}{2}(\nu=\psi, \gamma, t) .
$$

The term $\tilde{\psi}^{n+\frac{1}{2}}$ is actually the second-order extrapolation of $\psi$ at time $t=t^{n+\frac{1}{2}}$. For the initialization step in (2.13), $\psi^{1}$ is always evaluated by one step of a (implicit) CN scheme, which preserves the total mass and original total energy. For the conservative properties of the $\mathrm{CN}-\mathrm{AB}$ scheme, we first have the following result. 
Proposition 2.1. The $C N-A B$ scheme (2.13) preserves the following modified semi-discrete total energy

$$
\mathcal{E}_{\text {mod }}^{n}:=\mathcal{E}_{\text {mod }}\left(\psi^{n}\right)=\mathcal{E}_{0}\left(\psi^{n}\right)+\left|\gamma^{n}\right|^{2}=\mathcal{E}_{\text {mod }}^{0}=\mathcal{E}_{\text {tot }}\left(\psi_{0}\right)+\mathcal{E}_{c}
$$

with $\psi^{0}:=\psi_{0}$. Since the initial modified energy $\mathcal{E}_{\mathrm{mod}}^{0}=\left.\mathcal{E}_{\mathrm{tot}}\right|_{t=0}$, it implies that the modified energy preserves the initial energy of the system.

Proof. Let us consider the first equation of 2.13 that we multiply by $\left(\bar{\psi}^{n+1}-\bar{\psi}^{n}\right)$ and integrate over $\mathbb{R}^{d}$. Then, one gets

$$
\begin{aligned}
\frac{i}{\Delta t} \int_{\mathbb{R}^{d}}\left[\left|\psi^{n+1}\right|^{2}\right. & \left.+\left|\psi^{n}\right|^{2}-2 \operatorname{Re}\left(\psi^{n+1} \bar{\psi}^{n}\right)\right] d \mathbf{x} \\
= & \frac{1}{2} \int_{\mathbb{R}^{d}}\left[\left(\mathcal{L} \psi^{n+1}\right) \bar{\psi}^{n+1}-\left(\mathcal{L} \psi^{n}\right) \bar{\psi}^{n}-2 i \operatorname{Im}\left(\left(\mathcal{L} \psi^{n+1}\right) \bar{\psi}^{n}\right)\right] d \mathbf{x} \\
& +\int_{\mathbb{R}^{d}} \tilde{g}^{n+\frac{1}{2}} \gamma^{n+\frac{1}{2}}\left(\bar{\psi}^{n+1}-\bar{\psi}^{n}\right) d \mathbf{x} .
\end{aligned}
$$

Taking the real part of both sides of equation (2.15), we have

$$
\frac{1}{2}\left(\mathcal{E}_{0}\left(\psi^{n+1}\right)-\mathcal{E}_{0}\left(\psi^{n}\right)\right)+\gamma^{n+\frac{1}{2}} \int_{\mathbb{R}^{d}} \operatorname{Re}\left[\tilde{g}^{n+\frac{1}{2}}\left(\bar{\psi}^{n+1}-\bar{\psi}^{n}\right)\right] d \mathbf{x}=0
$$

Multiplying the second equation of (2.13) by $\gamma^{n+\frac{1}{2}}$ yields

$$
\frac{\left|\gamma^{n+1}\right|^{2}-\left|\gamma^{n}\right|^{2}}{2}=\gamma^{n+\frac{1}{2}} \int_{\mathbb{R}^{d}} \operatorname{Re}\left[\tilde{g}^{n+\frac{1}{2}}\left(\bar{\psi}^{n+1}-\bar{\psi}^{n}\right)\right] d \mathbf{x} .
$$

Injecting (2.17) into (2.16) leads to

$$
\mathcal{E}_{0}\left(\psi^{n+1}\right)+\left|\gamma^{n+1}\right|^{2}=\mathcal{E}_{0}\left(\psi^{n}\right)+\left|\gamma^{n}\right|^{2} .
$$

The proof is completed.

We remark that the original total energy is not conserved. Numerical results show that the scheme approximates the original total energy at third order. As for the total mass, we have the following result.

Proposition 2.2. The $C N-A B$ scheme (2.13) approximates the total mass at third order, i.e.

$$
\mathcal{N}^{n+1}=: \int_{\mathbb{R}^{d}}\left|\psi^{n+1}\right|^{2} d \mathbf{x} \equiv \int_{\mathbb{R}^{d}}\left|\psi^{n}\right|^{2} d \mathbf{x}+\mathcal{O}\left((\Delta t)^{3}\right)=\mathcal{N}^{n}+\mathcal{O}\left((\Delta t)^{3}\right) .
$$

Proof. Let us multiply the first equation of $(2.13)$ by $\left(\bar{\psi}^{n+1}+\bar{\psi}^{n}\right)$ and integrate on $\mathbb{R}^{d}$. Taking the imaginary part of the resulting expression, one gets

$$
\frac{1}{\Delta t} \int_{\mathbb{R}^{d}}\left(\left|\psi^{n+1}\right|^{2}-\left|\psi^{n}\right|^{2}\right) d \mathbf{x}=\gamma^{n+\frac{1}{2}} \int_{\mathbb{R}^{d}} \operatorname{Im}\left(\tilde{g}^{n+\frac{1}{2}}\left(\bar{\psi}^{n+1}+\bar{\psi}^{n}\right)\right) d \mathbf{x} .
$$

Noticing that $\psi^{n+1 / 2}=\tilde{\psi}^{n+1 / 2}+\mathcal{O}\left((\Delta t)^{2}\right)$, we have

$$
\tilde{g}^{n+\frac{1}{2}}:=g\left(\tilde{\psi}^{n+\frac{1}{2}}\right)=g\left(\psi^{n+\frac{1}{2}}\right)+\mathcal{O}\left((\Delta t)^{2}\right) .
$$


Plugging this relation into 2.20 and noticing that $\int_{\mathbb{R}^{d}} \operatorname{Im}(g(\psi) \bar{\psi}) d \mathbf{x}=0$ for all $\psi$, we obtain $\frac{1}{\Delta t} \int_{\mathbb{R}^{d}}\left(\left|\psi^{n+1}\right|^{2}-\left|\psi^{n}\right|^{2}\right) d \mathbf{x}=\gamma^{n+\frac{1}{2}} \int_{\mathbb{R}^{d}} \operatorname{Im}\left(g\left(\psi^{n+\frac{1}{2}}\right)\left(\bar{\psi}^{n+1}+\bar{\psi}^{n}\right)\right) d \mathbf{x}+\mathcal{O}\left((\Delta t)^{2}\right)=\mathcal{O}\left((\Delta t)^{2}\right)$,

which leads to the conclusion.

The linearly implicit semi-discrete scheme 2.13 is a coupled system for $\left(\psi^{n+1}, \gamma^{n+1}\right)$, since $\gamma^{n+1}$ is a scalar variable. We now show that it could actually be solved efficiently by using the decomposition

$$
\psi^{n+\frac{1}{2}}=\psi_{1}^{n+\frac{1}{2}}+\gamma^{n+\frac{1}{2}} \psi_{2}^{n+\frac{1}{2}} .
$$

Plugging (2.21) into the first equation of 2.13$)$, we find that

$$
\left\{\begin{array}{l}
\left(\frac{2 i}{\Delta t}-\mathcal{L}\right) \psi_{1}^{n+\frac{1}{2}}=\frac{2 i}{\Delta t} \psi^{n} \\
\left(\frac{2 i}{\Delta t}-\mathcal{L}\right) \psi_{2}^{n+\frac{1}{2}}=\tilde{g}^{n+\frac{1}{2}}
\end{array}\right.
$$

which can be written as

$$
\mathcal{A} \psi_{j}^{n+\frac{1}{2}}:=\left(\frac{2 i}{\Delta t}-\mathcal{L}\right) \psi_{j}^{n+\frac{1}{2}}=h_{j}^{n} \quad(j=1,2)
$$

where $h_{j}^{n}$ includes all known terms at previous steps. After solving $\psi_{j}^{n+\frac{1}{2}}(j=1,2)$ from (2.23), we plug (2.21) in the second equation of (2.13) to obtain

$$
\gamma^{n+\frac{1}{2}}=\frac{\gamma^{n}+\operatorname{Re}\left\langle\tilde{g}^{n+\frac{1}{2}}, \psi_{1}^{n+\frac{1}{2}}-\psi^{n}\right\rangle}{1-\operatorname{Re}\left\langle\tilde{g}^{n+\frac{1}{2}}, \psi_{2}^{n+\frac{1}{2}}\right\rangle}
$$

To summarize, the CN-AB scheme 2.13) can be efficiently implemented as follows:

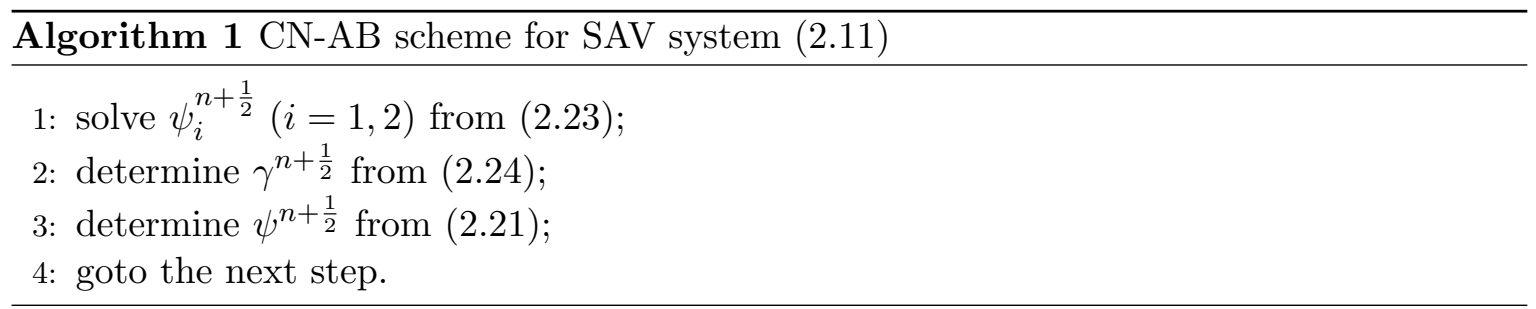

The main computational cost is to solve the two linear systems $(2.23)$ defined by the same $n$-independent operator, hence leading to a very efficient scheme. However, the scheme only conserves a modified total energy and approximates the total mass at third order. Below, we propose an efficient Lagrange multiplier approach which conserves both the mass and original total energy. 


\subsection{SAV via Lagrange multiplier approach}

To enforce the mass and energy conservations, inspired by the work in [20, 21], we introduce two Lagrange (Lag) multipliers $\eta_{1}(t)$ and $\eta_{2}(t)$, and rewrite (1.1) as the following Lag-GPE: for $\mathbf{x} \in \mathbb{R}^{d}, t \geq 0$,

$$
\left\{\begin{array}{l}
i \partial_{t} \psi(\mathbf{x}, t)=\mathcal{L} \psi+\eta_{1}(t) \frac{\delta \mathcal{E}_{1}}{\delta \bar{\psi}}+\eta_{2}(t) \frac{\delta \mathcal{N}}{\delta \psi} \\
\frac{d}{d t} \mathcal{E}_{1}(\psi(\cdot, t))=2 \eta_{1}(t) \operatorname{Re}\left\langle\frac{\delta \mathcal{E}_{1}}{\delta \bar{\psi}}, \partial_{t} \psi\right\rangle+2 \eta_{2}(t) \operatorname{Re}\left\langle\frac{\delta \mathcal{N}}{\delta \psi}, \partial_{t} \psi\right\rangle \\
\frac{d}{d t} \mathcal{N}(\psi(\cdot, t))=0
\end{array}\right.
$$

for $\psi_{0}$ given and $\eta_{1}(0)=1$ and $\eta_{2}(0)=0$. The exact solution of $\left(\eta_{1}(t), \eta_{2}(t)\right)$ is $(1,0)$, which means that the Lag-GPE system (2.25) is equivalent to the original GPE (1.1). Note that the last term in the second equation of $(2.25)$ is indeed zero thanks to the third equation of 2.25). The purpose to add this term is to construct an energy-conservative scheme later. It is clear that the third equation of 2.25 guarantees the mass conservation. Taking the inner product of the first equation of (2.25), considering real parts of the resulting equation, noticing (2.9) and using the second equation of 2.25), we obtain

$0=\operatorname{Re}\left\langle\frac{\delta \mathcal{E}_{0}}{\delta \bar{\psi}}, \partial_{t} \psi\right\rangle+\operatorname{Re}\left\langle\eta_{1}(t) \frac{\delta \mathcal{E}_{1}}{\delta \bar{\psi}}+\eta_{2}(t) \frac{\delta \mathcal{N}}{\delta \psi}, \partial_{t} \psi\right\rangle=\frac{1}{2} \frac{d}{d t}\left(\mathcal{E}_{0}(\psi)+\mathcal{E}_{1}(\psi)\right)=\frac{1}{2} \frac{d}{d t} \mathcal{E}_{\text {tot }}(t)$

which indicates that the original total energy $\mathcal{E}_{\text {tot }}$ given by 1.5 is preserved.

Standard semi-implicit schemes can again be used to discretize the Lag-GPE system 2.25). We propose to apply a second-order Crank-Nicolson Leap-Frog (CN-LF) scheme as follows: for $\mathbf{x} \in \mathbb{R}^{d}$ and $n \geq 1$

$$
\left\{\begin{array}{l}
i \frac{\psi^{n+1}-\psi^{n-1}}{2 \Delta t}=\mathcal{L} \frac{\psi^{n+1}+\psi^{n-1}}{2}+\eta_{1}^{n+1} \frac{\delta \mathcal{E}_{1}\left(\psi^{n}\right)}{\delta \overline{\psi^{n}}}+\eta_{2}^{n+1} \frac{\delta \mathcal{N}\left(\psi^{n}\right)}{\delta \psi^{n}} \\
\mathcal{E}_{1}\left(\psi^{n+1}\right)-\mathcal{E}_{1}\left(\psi^{n-1}\right)=2 \operatorname{Re}\left\langle\eta_{1}^{n+1} \frac{\delta \mathcal{E}_{1}\left(\psi^{n}\right)}{\delta \overline{\psi^{n}}}+\eta_{2}^{n+1} \frac{\delta \mathcal{N}\left(\psi^{n}\right)}{\delta \psi^{n}}, \psi^{n+1}-\psi^{n-1}\right\rangle \\
\mathcal{N}\left(\psi^{n+1}\right)=\mathcal{N}\left(\psi_{0}\right),
\end{array}\right.
$$

with $\psi^{0}=\psi_{0}$ and $\psi^{1}$ is evaluated by one step of a (implicit) $\mathrm{CN}$ scheme for initializing. For the conservative properties, we have the following result.

Proposition 2.3. The CN-LF scheme 2.26) preserves both the mass

$$
\mathcal{N}^{n}=: \mathcal{N}\left(\psi^{n}\right)=\mathcal{N}^{0}, \quad n \geq 1,
$$

and the original total energy

$$
\mathcal{E}_{\text {tot }}^{n}=: \mathcal{E}_{\text {tot }}\left(\psi^{n}\right)=\mathcal{E}_{\text {tot }}^{0}, \quad n \geq 1 .
$$


Proof. The third equation in (2.26) guarantees the conservation of the mass. As for the total energy, by multiplying the first equation in $(2.26)$ by $\bar{\psi}^{n+1}-\bar{\psi}^{n}$ and integrating over $\mathbb{R}^{d}$, we obtain

$$
\begin{aligned}
\frac{i}{2 \Delta t} \int_{\mathbb{R}^{d}}\left[\left|\psi^{n+1}\right|^{2}\right. & \left.+\left|\psi^{n-1}\right|^{2}-2 \operatorname{Re}\left(\psi^{n+1} \bar{\psi}^{n-1}\right)\right] d \mathbf{x} \\
= & \frac{1}{2}\left[\left\langle\mathcal{L} \psi^{n+1}, \psi^{n+1}\right\rangle-\left\langle\mathcal{L} \psi^{n-1}, \psi^{n-1}\right\rangle-2 i \operatorname{Im}\left\langle\mathcal{L} \psi^{n+1}, \psi^{n-1}\right\rangle\right] \\
& +\left\langle\eta_{1}^{n+1} \frac{\delta \mathcal{E}_{1}\left(\psi^{n}\right)}{\delta \overline{\psi^{n}}}+\eta_{2}^{n+1} \frac{\delta \mathcal{N}\left(\psi^{n}\right)}{\delta \psi^{n}}, \psi^{n+1}-\psi^{n-1}\right\rangle .
\end{aligned}
$$

Taking the real parts of both sizes of the equation 2.29 and noticing the second equation of $(2.26)$, we have

$$
\mathcal{E}_{0}\left(\psi^{n+1}\right)-\mathcal{E}_{0}\left(\psi^{n-1}\right)+\mathcal{E}_{1}\left(\psi^{n+1}\right)-\mathcal{E}_{1}\left(\psi^{n-1}\right)=0
$$

which yields

$$
\mathcal{E}_{\text {tot }}\left(\psi^{n+1}\right)=\mathcal{E}_{0}\left(\psi^{n+1}\right)+\mathcal{E}_{1}\left(\psi^{n+1}\right)=\mathcal{E}_{0}\left(\psi^{n-1}\right)+\mathcal{E}_{1}\left(\psi^{n-1}\right)=\mathcal{E}_{\text {tot }}\left(\psi^{n-1}\right) .
$$

Since $\psi^{1}$ is evaluated by the standard (implicit) CN scheme, the mass and original total energy is preserved for $\psi^{1}$. Hence, the original total energy is conserved for all $n \geq 0$, ending hence the proof.

Similarly to the CN-AB scheme (2.13), the CN-LP scheme 2.26$)$ could be solved efficiently as follows. Writing

$$
\psi^{n+1}=\psi_{1}^{n+1}+\eta_{1}^{n+1} \psi_{2}^{n+1}+\eta_{2}^{n+1} \psi_{3}^{n+1},
$$

and plugging the expression into the first equation of (2.26), by a simple calculation, we obtain

$$
\left\{\begin{array}{l}
\left(\frac{i}{\Delta t}-\mathcal{L}\right) \psi_{1}^{n+1}=\left(\frac{i}{\Delta t}+\mathcal{L}\right) \psi^{n-1} \\
\left(\frac{i}{\Delta t}-\mathcal{L}\right) \psi_{2}^{n+1}=2 \frac{\delta \mathcal{E}_{1}\left(\psi^{n}\right)}{\delta \overline{\psi^{n}}} \\
\left(\frac{i}{\Delta t}-\mathcal{L}\right) \psi_{3}^{n+1}=2 \frac{\delta \mathcal{N}\left(\psi^{n}\right)}{\delta \psi^{n}}
\end{array}\right.
$$

which can be written as

$$
\widetilde{\mathcal{A}} \psi_{j}^{n+1}:=\left(\frac{i}{\Delta t}-\mathcal{L}\right) \psi_{j}^{n+1}=\tilde{h}_{j}^{n} \quad(j=1,2,3),
$$

where $\tilde{h}_{j}^{n}$ includes all known terms at previous steps. After solving $\psi_{j}^{n+1}(j=1,2,3)$ from (2.34), we plug (2.32) into the second and third equation of (2.26), leading to a coupled nonlinear $2 \times 2$ algebraic system for $\left(\eta_{1}^{n+1}, \eta_{2}^{n+1}\right)$ :

$$
\left\{\begin{array}{l}
\mathcal{F}_{1}\left(\eta_{1}^{n+1}, \eta_{2}^{n+1}\right)=0, \\
\mathcal{F}_{2}\left(\eta_{1}^{n+1}, \eta_{2}^{n+1}\right)=0
\end{array}\right.
$$


where the nonlinear function $\mathcal{F}_{\ell}\left(\eta_{1}, \eta_{2}\right)(\ell=1,2)$ reads as

$$
\begin{aligned}
& \mathcal{F}_{1}\left(\eta_{1}, \eta_{2}\right)=\mathcal{E}_{1}\left(\psi_{\eta}^{n+1}\right)-\mathcal{E}_{1}\left(\psi^{n-1}\right)-2 \operatorname{Re}\left\langle\eta_{1} \frac{\delta \mathcal{E}_{1}\left(\psi^{n}\right)}{\delta \overline{\psi^{n}}}+\eta_{2} \frac{\delta \mathcal{N}\left(\psi^{n}\right)}{\delta \psi^{n}}, \psi_{\eta}^{n+1}-\psi^{n-1}\right\rangle, \\
& \mathcal{F}_{2}\left(\eta_{1}, \eta_{2}\right)=\mathcal{N}\left(\psi_{\eta}^{n+1}\right)-\mathcal{N}\left(\psi_{0}\right), \quad \psi_{\eta}^{n+1}:=\eta_{1} \psi_{2}^{n+1}+\eta_{2} \psi_{3}^{n+1}+\psi_{1}^{n+1} .
\end{aligned}
$$

To summarize, the scheme 2.26 can be efficiently implemented as follows:

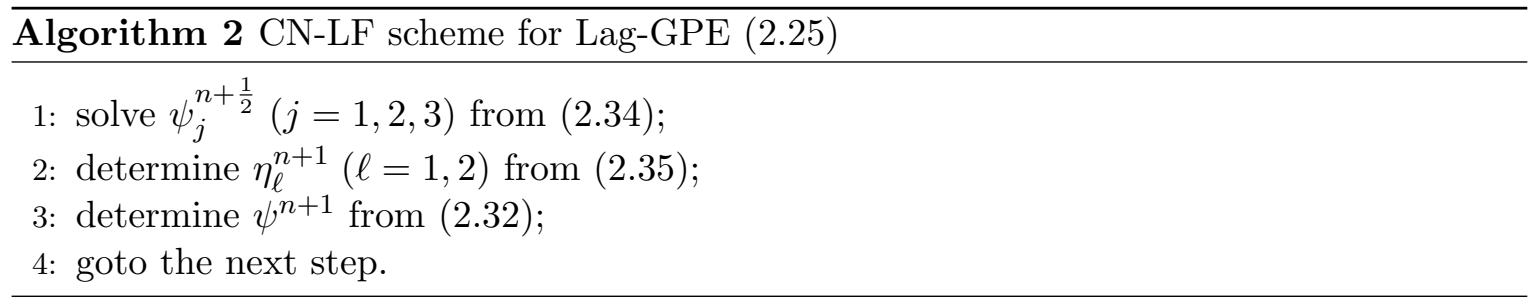

The complexity and solvability of the system (2.35) depends on the structure of the energy splitting $\mathcal{E}_{1}(\psi)$. When it is solvable (i.e., the real roots exist), it can be solved by (descent) Newton iteration efficiently. Since the exact solution for $\left(\eta_{1}(t), \eta_{2}(t)\right)$ is $(1,0)$, we can use $(1,0)$ as the initial guess for the Newton iteration. With this initial guess, the Newton iteration would usually converges quickly. Therefore, the main computational cost remain in solving the three linear systems $(2.33)$ with $n$-independent operator, leading to a efficient scheme.

\subsection{Two typical choices of energy splitting}

We consider first the usual energy splitting strategy which is essentially splitting the total energy into a linear and a nonlinear parts as follows

$$
\begin{aligned}
\mathcal{E}_{\mathrm{tot}}(\psi) & =\int_{\mathbb{R}^{d}}\left[\frac{1}{2}|\nabla \psi|^{2}+V(\mathbf{x})|\psi|^{2}-\omega \operatorname{Re}\left(\bar{\psi} L_{z} \psi\right)\right] d \mathbf{x}+\int_{\mathbb{R}^{d}}\left[F(\rho)+\frac{\lambda}{2}|\nabla \rho|^{2}\right] d \mathbf{x} \\
& :=\mathcal{E}_{0}(\psi)+\mathcal{E}_{1}(\psi) .
\end{aligned}
$$

It is clear that $\mathcal{E}_{1}(\psi) \geq 0$ if $F(\rho)>0$ (which is true for most cases in context of BEC), and therefore we can choose any constant $\mathcal{E}_{c}>0$. In this case, one gets

$$
\mathcal{L} \psi=\frac{\delta \mathcal{E}_{0}(\psi)}{\delta \bar{\psi}}=\left(-\frac{\nabla^{2}}{2}+V(\mathbf{x})-\omega L_{z}\right) \psi
$$

Hence, the linear operator $\mathcal{A}=(2 i / \Delta t-\mathcal{L})$ in $(2.23)$ and $\widetilde{\mathcal{A}}=(i / \Delta t-\mathcal{L})$ in $(2.34)$ are fixed operators that do not depend on $n$. This is an important practical point since, when discretizing in space, the matrix representation only needs to be computed once before entering into the time loop as well as its LU factorization when using finite difference or finite element, making the scheme very efficient and with a low memory requirement. However, since $\mathcal{A}$ and $\widetilde{\mathcal{A}}$ involve variable coefficients, it is not easily invertible with a spectral discretization in space. Therefore, we propose to consider another energy splitting leading to operators $\mathcal{A}$ and $\widetilde{\mathcal{A}}$ with constant coefficients, which is therefore more adapted to a spectral discretization in space. 
To this end, we split the energy functional 1.5 as the sum of the two following energies

$$
\begin{aligned}
\mathcal{E}_{\mathrm{tot}}(t)= & \int_{\mathbb{R}^{d}}\left[\left(\frac{1}{2}+C_{\mathrm{HOI}}\right)|\nabla \psi|^{2}+W|\psi|^{2}\right] d \mathbf{x} \\
& +\int_{\mathbb{R}^{d}}\left[(V(\mathbf{x})-W)|\psi|^{2}-\omega \operatorname{Re}\left(\bar{\psi} L_{z} \psi\right)+F(\rho)-C_{\mathrm{HOI}}|\nabla \psi|^{2}+\frac{\lambda}{2}|\nabla \rho|^{2}\right] d \mathbf{x} \\
= & \mathcal{E}_{0}(\psi(\cdot, t))+\mathcal{E}_{1}(\psi(\cdot, t)) .
\end{aligned}
$$

Here, the two positive constants $W>0$ and $C_{\mathrm{HOI}}>0$ are suitable stabilization parameters to properly counterbalance the translated potential, the lower-order interaction and the HOI terms, respectively. With this splitting, it is easy to check that

$$
\begin{aligned}
& \mathcal{L} \psi=\frac{\delta \mathcal{E}_{0}(\psi)}{\delta \bar{\psi}}=-\left(\left(\frac{1}{2}+C_{\mathrm{HOI}}\right) \nabla^{2}-W\right) \psi \\
& \frac{\delta \mathcal{E}_{1}(\psi)}{\delta \bar{\psi}}=\left(V(\mathbf{x})-W-\omega L_{z}+f(\rho)+C_{\mathrm{HOI}} \nabla^{2}-\lambda \nabla^{2} \rho\right) \psi
\end{aligned}
$$

Hence, both $\mathcal{A}$ and $\widetilde{\mathcal{A}}$ are $n$-independent operators with constant coefficients. Thus, systems (2.23) and (2.34) could be efficiently straightforwardly solved with a spectral discretization. In particular, for the Fourier-spectral method considered in this paper, $\mathcal{A}$ and $\widetilde{\mathcal{A}}$ lead to diagonal matrices. The linear implicit form of the CN-AB and CN-LF schemes is a crucial difference compared for example with the usual CN scheme which requires the use of a nonlinear solver at each time step or the relaxation scheme which leads to solve operator equations which depend on $n$ (since the nonlinearity is updated at each time step).

Remark 2.1. The expression (2.37) indicates that $\mathcal{E}_{0} \geq 0$. Now that the total energy $\mathcal{E}_{\text {tot }}$ is conserved, we clearly see that

$$
\mathcal{E}_{1}(\phi(\cdot, t))=\mathcal{E}_{\text {tot }}(\psi(\cdot, t))-\mathcal{E}_{0}(\psi(\cdot, t)) \geq \mathcal{E}_{\text {tot }}\left(\psi_{0}(\mathbf{x})\right)
$$

is lower bounded. Hence, the constant $\mathcal{E}_{c}$ always exists for the SAV approach. Stabilization terms related to $W$ and $C_{\mathrm{HOI}}$ can greatly enhance the performance of both the $C N-A B$ and $C N-L F$ schemes. Without proper stabilization, the schemes would require a much smaller time step $\Delta t$ regarding the nonlinear and potential terms. On the other hand, the current simple stabilization does not consider the rotation term. We will see during the numerical simulations that $\Delta t$ must be taken smaller as the rotation speed increases.

\subsection{Pseudospectral discretization}

We truncate the problem (1.1) as a bounded domain $\mathcal{D}$ with periodic boundary condition at the boundary $\partial \mathcal{D}$. To simplify the presentation, we only describe the schemes in $2 \mathrm{D}$, their extensions to other dimensions being straightforward. Now that we would like to apply the Fourier pseudospectral method [2, 5, 7] for the spatial discretization, we choose the second energy-splitting (2.37) for both the CN-AB 2.13) and CN-LF (2.26) schemes. Let us define the square domain $\mathcal{D}=\left[-L_{x} ; L_{x}\right] \times\left[-L_{y} ; L_{y}\right]$. Let $h_{x}=\frac{2 L_{x}}{L}$ and $h_{y}=\frac{2 L_{y}}{M}$ be the uniform 
mesh sizes in the $x$ - and $y$-directions, where $L$ and $M$ are two even integers, respectively. To simplify the notations, we define the indices, grid points sets and basis functions as

$$
\begin{aligned}
\mathcal{T}_{L M} & =\left\{(\ell, m) \in \mathbb{N}^{2} \mid 0 \leq \ell \leq L, 0 \leq m \leq M\right\}, \\
\widetilde{\mathcal{T}}_{L M} & =\left\{(p, q) \in \mathbb{Z}^{2} \mid-L / 2 \leq p \leq L / 2-1,-M / 2 \leq q \leq M / 2-1\right\}, \\
\mathcal{G}_{x y} & =\left\{\left(x_{\ell}, y_{m}\right):=\left(-L_{x}+\ell h_{x},-L_{y}+m h_{y}\right),(\ell, m) \in \mathcal{T}_{L M}\right\}, \\
W_{p q}(\mathbf{x}) & =e^{i \mu_{p}^{x}\left(x+L_{x}\right)} e^{i \mu_{q}^{y}\left(y+L_{y}\right)}, \quad \mu_{p}^{x}=\pi p / L_{x}, \mu_{q}^{y}=\pi q / L_{y}, \quad(p, q) \in \widetilde{\mathcal{T}}_{L M} .
\end{aligned}
$$

Let us denote by $\psi_{\ell m}^{n}$ the approximation of $\psi\left(x_{\ell}, y_{m}, t_{n}\right)$ for $(\ell, m) \in \mathcal{T}_{L M}$ and $n \geq 0$. We define $\boldsymbol{\psi}^{n}$ as the vector with components $\left\{\psi_{\ell m}^{n}\right\}_{(\ell, m) \in \mathcal{T}_{L M}}$. The Fourier pseudospectral discretization of a given function $\psi$ is then given by

$$
\psi(x, y, t)=\sum_{p=-L / 2}^{L / 2-1} \sum_{q=-M / 2}^{M / 2-1} \widehat{\psi}_{p q}(t) W_{p q}(x, y)
$$

where the Fourier coefficients $\widehat{\psi}_{p q}(t)$ are such that

$$
\widehat{\psi}_{p q}(t)=\frac{1}{L M} \sum_{j=0}^{L-1} \sum_{k=0}^{M-1} \psi_{\ell m}(t) e^{-i \mu_{p}^{x}\left(x_{\ell}+L_{x}\right)} e^{-i \mu_{q}^{y}\left(y_{m}+L_{y}\right)} .
$$

Therefore, the Fourier pseudospectral discretizations of the derivative operators $\partial_{x}^{k} \psi$ and $\partial_{y}^{k} \psi$, for $k=1,2$, are respectively given by: for $(\ell, m) \in \mathcal{T}_{L M}$

$$
\begin{aligned}
& \left(\llbracket \partial_{x}^{k} \rrbracket \psi\right)_{\ell m}(t)=\sum_{p=-L / 2}^{L / 2-1} \sum_{q=-M / 2}^{M / 2-1}\left(i \mu_{p}^{x}\right)^{k} \widehat{\psi}_{p q}(t) W_{p q}\left(x_{\ell}, y_{m}\right), \\
& \left(\llbracket \partial_{y}^{k} \rrbracket \psi\right)_{\ell m}(t)=\sum_{p=-L / 2}^{L / 2-1} \sum_{q=-M / 2}^{M / 2-1}\left(i \mu_{q}^{y}\right)^{k} \widehat{\psi}_{p q}(t) W_{p q}\left(x_{\ell}, y_{m}\right),
\end{aligned}
$$

and $\llbracket \nabla^{2} \rrbracket:=\llbracket \partial_{x}^{2} \rrbracket+\llbracket \partial_{y}^{2} \rrbracket, \llbracket \nabla \rrbracket:=\left(\llbracket \partial_{x} \rrbracket, \llbracket \partial_{y} \rrbracket\right)^{t}$. In addition, we define the discrete operators $\mathbb{L}, \mathbb{A}, \widetilde{\mathbb{A}}, \mathbb{V}, \mathbb{W}$ and $\mathcal{L}_{z}$ as follows

$$
\begin{aligned}
& \mathbb{L}:=\left(1 / 2+C_{\mathrm{HOI}}\right) \llbracket \nabla^{2} \rrbracket-\mathbb{W}, \quad \mathbb{A}:=\frac{2 i}{\Delta t} \mathbb{I}-\mathbb{L}, \quad \widetilde{\mathbb{A}}:=\frac{i}{\Delta t} \mathbb{I}-\mathbb{L}, \\
& (\mathbb{V} \boldsymbol{\psi})_{\ell m}=V_{\ell m} \psi_{\ell m}, \quad(\mathbb{W} \boldsymbol{\psi})_{\ell m}=W \psi_{\ell m}, \\
& \left(\mathcal{L}_{z} \boldsymbol{\psi}\right)_{\ell m}=i\left(y_{m}\left(\llbracket \partial_{x} \boldsymbol{\psi} \rrbracket\right)_{\ell m}-x_{\ell}\left(\llbracket \partial_{y} \boldsymbol{\psi} \rrbracket\right)_{\ell m}\right),
\end{aligned}
$$

for $(\ell, m) \in \mathcal{T}_{L M}$, where $\mathbb{I}$ denotes the identity matrix. Note that the operators $\mathbb{A}$ and $\widetilde{\mathbb{A}}$ are all diagonal in Fourier space since $W$ is a constant, thus they could be easily inverted and (2.13) and (2.26) could be efficiently solved by two FFT operations (one FFT followed by one inverse FFT).

Based on these notations, then the pseudospectral CN-AB (CN-AB-SP) scheme yields Algorithm 3. Moreover, the pseudospectral CN-LF (CN-LF-SP) scheme yields Algorithm 

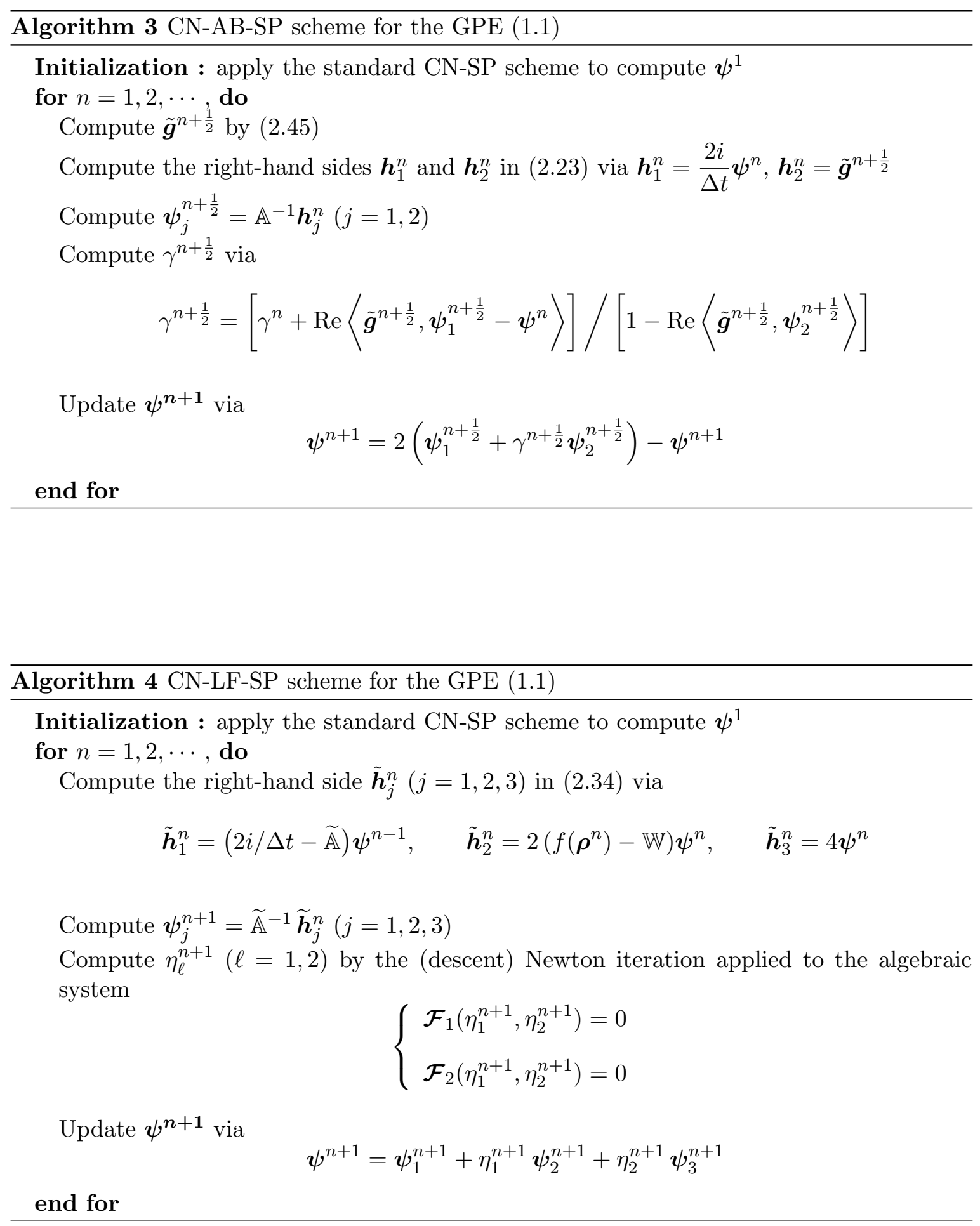
4. Here, CN-SP means Crank-Nicolson scheme with pseudospectral approximation in space [2, 5].

In the above algorithms, we introduced the following notations

$$
\begin{aligned}
& \tilde{\boldsymbol{\psi}}^{n+\frac{1}{2}}=\frac{3}{2} \boldsymbol{\psi}^{n}-\frac{1}{2} \boldsymbol{\psi}^{n-1}, \quad \tilde{\boldsymbol{g}}^{n+\frac{1}{2}}=\boldsymbol{g}\left(\tilde{\boldsymbol{\psi}}^{n+\frac{1}{2}}\right), \quad \boldsymbol{\nu}^{n+\frac{1}{2}}=\frac{\boldsymbol{\nu}^{n+1}+\boldsymbol{\nu}^{n}}{2}(\nu=\boldsymbol{\psi}, \gamma), \\
& \boldsymbol{g}(\boldsymbol{\psi}):=\left[\left(f(\boldsymbol{\rho})+(\mathbb{V}(\mathbf{x})-\mathbb{W})-\lambda \llbracket \nabla^{2} \rrbracket \boldsymbol{\rho}\right) \boldsymbol{\psi}-\omega \mathcal{L}_{z} \boldsymbol{\psi}\right] / \sqrt{\mathcal{E}_{1}(\boldsymbol{\psi})+\mathcal{E}_{c}} \\
& \mathcal{F}_{1}\left(\eta_{1}, \eta_{2}\right)=2 \operatorname{Re}\left\langle\eta_{1}\left(f\left(\boldsymbol{\rho}^{n}\right)-\mathbb{W}\right) \boldsymbol{\psi}^{n}+2 \eta_{2} \boldsymbol{\psi}^{n}, \boldsymbol{\psi}_{\eta}^{n+1}-\boldsymbol{\psi}^{n-1}\right\rangle-\mathcal{E}_{1}\left(\boldsymbol{\psi}_{\eta}^{n+1}\right)+\mathcal{E}_{1}\left(\boldsymbol{\psi}^{n-1}\right), \\
& \mathcal{F}_{2}\left(\eta_{1}, \eta_{2}\right)=\mathcal{N}\left(\boldsymbol{\psi}_{\eta}^{n+1}\right)-\mathcal{N}\left(\boldsymbol{\psi}_{0}\right), \quad \boldsymbol{\psi}_{\eta}^{n+1}:=\eta_{1} \boldsymbol{\psi}_{2}^{n+1}+\eta_{2} \boldsymbol{\psi}^{n+1}+\boldsymbol{\psi}_{1}^{n+1} .
\end{aligned}
$$

Here, the multiplication between two vectors $\boldsymbol{v}$ and $\boldsymbol{w}$ is made pointwise, i.e., setting $\boldsymbol{z}:=$ $\boldsymbol{v} \boldsymbol{w}$, for $(\boldsymbol{z})_{\ell m}:=v_{\ell m} w_{\ell m}$ on the spatial grid. The vector $\boldsymbol{\rho}$ is then defined by $\boldsymbol{\rho}:=\boldsymbol{\psi} \overline{\boldsymbol{\psi}}$. In addition, the inner product and discrete 2-norm are respectively defined as $\langle\boldsymbol{v}, \boldsymbol{w}\rangle=$ : $h_{x} h_{y} \boldsymbol{v} \overline{\boldsymbol{w}}$, and $\|\boldsymbol{v}\|_{2}:=\langle\boldsymbol{v}, \boldsymbol{v}\rangle^{1 / 2}$. The discrete mass and energies are therefore given by

$$
\begin{aligned}
& \mathcal{N}\left(\boldsymbol{\psi}^{n}\right):=\left\|\boldsymbol{\psi}^{n}\right\|_{2}^{2}, \quad \mathcal{E}_{\text {kin }}\left(\boldsymbol{\psi}^{n}\right):=\frac{1}{2}\left\|\llbracket \nabla \rrbracket \boldsymbol{\psi}^{n}\right\|_{2}^{2}, \quad \mathcal{E}_{\mathrm{pot}}^{\mathbb{V}}\left(\boldsymbol{\psi}^{n}\right):=\left\langle\mathbb{V} \boldsymbol{\psi}^{n}, \boldsymbol{\psi}^{n}\right\rangle, \\
& \mathcal{E}_{\text {int }}\left(\boldsymbol{\psi}^{n}\right):=\left\langle F\left(\boldsymbol{\rho}^{n}\right), \mathbf{1}\right\rangle+\frac{\lambda}{2}\left\|\llbracket \nabla \rrbracket \boldsymbol{\rho}^{n}\right\|_{2}^{2}, \quad \mathcal{E}_{\mathrm{rot}}\left(\boldsymbol{\psi}^{n}\right)=-\omega\left\langle\mathcal{L}_{z} \boldsymbol{\psi}^{n}, \boldsymbol{\psi}^{n}\right\rangle, \\
& \mathcal{E}_{1}\left(\boldsymbol{\psi}^{n}\right)=\mathcal{E}_{\mathrm{pot}}^{\mathbb{V}-\mathbb{W}}\left(\boldsymbol{\psi}^{n}\right)+\mathcal{E}_{\text {rot }}\left(\boldsymbol{\psi}^{n}\right)+\mathcal{E}_{\text {int }}\left(\boldsymbol{\psi}^{n}\right), \\
& \mathcal{E}_{\text {tot }}\left(\boldsymbol{\psi}^{n}\right)=\mathcal{E}_{\text {kin }}\left(\boldsymbol{\psi}^{n}\right)+\mathcal{E}_{\text {pot }}^{\mathbb{V}}\left(\boldsymbol{\psi}^{n}\right)+\mathcal{E}_{\text {rot }}\left(\boldsymbol{\psi}^{n}\right)+\mathcal{E}_{\text {int }}\left(\boldsymbol{\psi}^{n}\right), \\
& \mathcal{E}_{\text {mod }}\left(\boldsymbol{\psi}^{n}\right)=\mathcal{E}_{\text {kin }}\left(\boldsymbol{\psi}^{n}\right)+\mathcal{E}_{\text {pot }}\left(\boldsymbol{\psi}^{n}\right)+\mathcal{E}_{\text {rot }}\left(\boldsymbol{\psi}^{n}\right)+\left|\gamma^{n}\right|^{2} .
\end{aligned}
$$

The CN-AB-SP (CN-LF-SP) scheme requires twice (thrice) the solution to a linear system defined by a time independent linear operator $\mathbb{A}(\widetilde{\mathbb{A}})$ at each time step $n$. When using a finite-difference or finite element discretization, then $\mathbb{A}$ and $\widetilde{\mathbb{A}}$ are given explicitly by a matrix and could be LU factorized before entering in the loop on $n$. For the pseudospectral approximation, as mentioned previously, the inversion is direct, leading then to a cost per iteration of order $\mathcal{O}(N \log N)$, where $N=L M$ in $2 \mathrm{D}$.

\section{Numerical results}

In this section, we present the performance of the two schemes for solving the NLSE/GPE for various $1 \mathrm{D}$ and $2 \mathrm{D}$ configurations. We first compare the two schemes to show their advantages and disadvantages. We then mainly investigate how well the CN-AB-SP scheme solves NLSE/GPE with some frequently-used nonlinearities by considering its order of accuracy and analyzing its properties related to the mass and total energy. Finally, we apply the CNAB-SP scheme to simulate some interesting phenomena in some physical systems, such as the bright soliton-kink interaction in (non-Kerr) optical media, vortex dynamics in rotating 
BEC. To quantify the numerical errors on the solution, mass and energy, we introduce the error functions

$$
\begin{aligned}
& e_{\infty}\left(t_{n}\right)=\left\|\boldsymbol{\psi}\left(\cdot, t_{n}\right)-\boldsymbol{\psi}^{n}\right\|_{\ell^{\infty}}, \quad e_{2}\left(t_{n}\right)=\left\|\boldsymbol{\psi}\left(\cdot, t_{n}\right)-\boldsymbol{\psi}^{n}\right\|_{\ell^{2}}, \quad e_{\mathcal{N}}\left(t_{n}\right)=\left|\mathcal{N}\left(\boldsymbol{\psi}^{n}\right)-\mathcal{N}\left(\boldsymbol{\psi}^{0}\right)\right|, \\
& e_{\mathcal{E}_{\mathrm{tot}}}\left(t_{n}\right)=\left|\mathcal{E}_{\mathrm{tot}}\left(\boldsymbol{\psi}^{n}\right)-\mathcal{E}_{\mathrm{tot}}\left(\boldsymbol{\psi}^{0}\right)\right|, \quad e_{\mathcal{E}_{\text {mod }}}^{\text {rel }}\left(t_{n}\right)=\left|\mathcal{E}_{\text {mod }}\left(\boldsymbol{\psi}^{n}\right)-\mathcal{E}_{\text {mod }}\left(\boldsymbol{\psi}^{0}\right)\right| /\left|\mathcal{E}_{\text {mod }}\left(\boldsymbol{\psi}^{0}\right)\right|,
\end{aligned}
$$

where $\|\cdot\|_{\ell^{2}}$ and $\|\cdot\|_{\ell^{\infty}}$ designate the discrete 2-norm and infinity norm, respectively. In the following, unless specified, we use the second energy-splitting (2.37) and choose the stabilization parameters $C_{\mathrm{HOI}}$ and $W$ as

$$
C_{\mathrm{HOI}}=|\lambda| / 2, \quad W:=\max _{\mathbf{x} \in \Omega} V(\mathbf{x})+\frac{|\beta|}{2}+c_{1}, \quad E_{c}:=W+C_{\mathrm{HOI}}+|\beta|+|\lambda|+c_{2} .
$$

Here, $c_{1}$ and $c_{2}$ are two constants that has to be properly chosen case by case.

\subsection{One-dimensional case}

In this section, we carry out some numerical results for the one-dimensional case, i.e. $d=1$, where there is no rotational term in (1.1). We first compare the CN-AB-SP and CN-LF-SP schemes and then focus on the performance of the CN-AB-SP scheme. To this end, unless specified, the computational domain and mesh size are chosen respectively as $\mathcal{D}=[-32,32]$ and $h_{x}=1 / 32$.

Example 3.1. Here, we compare the performance of the CN-AB-SP and CN-LF-SP schemes. To this end, we simulate the dynamics of a BEC without HOI, i.e., we take $f\left(|\psi|^{2}\right)=\beta|\psi|^{2}$, $\lambda=0, V(x)=x^{2} / 2$. The initial data is chosen as $\psi_{0}(x)=\phi_{\mathrm{g}}^{\beta}(x)$, where $\phi_{\mathrm{g}}^{\beta}(x)$ is the ground state of the GPE with the same parameters values as those considered in the dynamics. We fix $\Delta t=10^{-3}$. Figure 3.1 shows the time evolution of $e_{\mathcal{N}}, e_{\mathcal{E}_{\text {tot }}}$ and $e_{\infty}$ as well as the Lagrange multipliers $\eta_{j}(t)(j=1,2)$ and SAV parameter $\gamma(t)$ for different values of the nonlinearity strenght $\beta$. We can clearly see that the CN-LF-SP scheme for the Lag-GPE formulation preserves well the total mass and original total energy while the CN-AB-SP scheme does not for both quantities. Moreover, the Lag-GPE formulation provides a much more accurate solution than the CN-AB-SP scheme for the standard SAV formulation. The Lagrange multipliers $\left(\eta_{1}(t), \eta_{2}(t)\right)$ are close to the exact one $(1,0)$. The performances are similar for simulating the standing soliton in focusing and logarithmic NLSE (i.e. for $v=0$ in Cases 1 and 3 respectively in next example), which evidence the powerfulness and effectiveness of the CN-LF-SP scheme based on the Lagrange multiplier approach. Although the Lagrange multiplier approach outperforms the SAV approach when it works, it remains unclear on how to choose properly the stabilization parameter $W$ to ensure the existence of the real root of the algebraic system 2.35 which is close to $(1,0)$. We leave this as future work and in subsequent examples we simply apply the standard SAV approach.

Remark 3.1. Actually, whether or not the CN-LF-SP works depends heavily on the existence of the real roots of the algebraic system 2.35). In principle the nonlinear algebraic system should admit real roots close to the exact solution as $\Delta t$ becomes sufficiently small, since the CN-LF-SP is a consistent approximation to the original PDE. But how small should $\Delta t$ 
be to guarantee the existence of real roots close to the exact solution is indeed case-dependent and related to choices such as the stabilization parameter $W$. For example, we consider a moving soliton case in focusing NLSE (i.e. take $v=1$ in Cases 1 in next example). Without stabilization parameter, i.e. for $W=0$, we plot in Figure 3.2 the curves of $\mathcal{F}_{\ell}\left(\eta_{1}, \eta_{2}\right)=0$ $(\ell=1,2)$ for the algebraic system (2.35) at time $t=2 \Delta t$ (i.e., the first step to evolve CN$L F$-SP scheme). Clearly we can see there are no intersection of the two curves close to $(1,0)$, which implies the nonexistence of the real roots close to $(1,0)$ for the range of $\Delta t$ considered. On the other hand, when a stabilized term is added (i.e. $W \neq 0$ ), there would be real roots close to $(1,0)$ at $t=2 \Delta t$.

Example 3.2. Here, we consider the NLSE with various nonlinearities and the CN-AB-SP scheme. To this end, we fix $V(x)=0$ and $\lambda=0$ in (1.1) and study the following three nonlinearities:

- Case 1. The cubic nonlinearity, i.e. $f\left(|\psi|^{2}\right)=\beta|\psi|^{2}$, with $\beta<0$.

- Case 2. The cubic-quintic nonlinearity, i.e. $f\left(|\psi|^{2}\right)=\alpha_{1}|\psi|^{2}-\alpha_{2}|\psi|^{4}$, with $\alpha_{1}>0$ and $\alpha_{2}>0$.

- Case 3. The logarithmic nonlinearity, i.e. $f\left(|\psi|^{2}\right)=\beta \ln |\psi|^{2}$, with $\beta<0$.

All the three cases admit analytic soliton solutions [10, 14, 35] as

$$
\psi_{\text {sol }}(x, t)= \begin{cases}\frac{a}{\sqrt{-\beta}} \operatorname{sech}(a(x-v t)) e^{i v x-0.5\left(v^{2}-a^{2}\right) t}, & \text { Case 1, } \\ \sqrt{\frac{4 \alpha_{1} w / \alpha_{2}}{1+\sqrt{1-\frac{w}{w_{0}}} \cosh \left(2 \sqrt{w} \alpha x-\sqrt{w} \alpha^{2} v t\right)} e^{i\left(0.5 v \alpha(x-0.25 v \alpha t)+0.5 w \alpha^{2} t+\phi_{0}\right)},} & \text { Case 2, } \\ b e^{\beta(\sqrt{2} x-2 v t)^{2} / 2+i\left(\sqrt{2} v x-\left(\phi_{b}+v^{2}\right) t\right)}, & \text { Case 3, }\end{cases}
$$

if the initial data is chosen as $\psi_{0}(x)=\psi_{\text {sol }}(x, 0)$. In the above formulas, $a, w, v, x_{0}, b$ are constants and $\phi_{b}=\beta\left(\ln |b|^{2}-1\right), \alpha=\sqrt{2 \alpha_{1}^{2} / \alpha_{2}}, 0<w<w_{0}=3 / 16$. In the following simulations, we take $\beta=-1, \alpha_{1}=\alpha_{2}=50, a=1, b=\sqrt[4]{-\beta / \pi} w=w_{0} / 2, \phi_{0}=0$, and let $v=1$ for Cases 1 and $\mathbf{3}$ while $v=0.1$ for Case 2. Notice that for Case 3, we need to regularize the logarithmic nonlinearity first to overcome its singularity at the origin. Following [10, we use the regularization $f\left(|\psi|^{2}\right) \approx f^{\varepsilon}\left(|\psi|^{2}\right)=\beta \ln \left((\varepsilon+|\psi|)^{2}\right)$. We fix $\varepsilon=10^{-14}$ which is small enough so that the error arising from this regularization could be omitted.

For Cases 1-3, Figure 3.3 shows the errors $e_{2}(t), e_{\mathcal{N}}(t)$ and $e_{\mathcal{E}_{\text {tot }}}(t)$ vs the time step $\Delta t$ at $t=10$, while Figure 3.4 depicts the time evolution of the errors on the mass (i.e. $e_{\mathcal{N}}(t)$ ), on the original total energy (i.e. $\left.e_{\mathcal{E}_{\text {tot }}}(t)\right)$ and on the modified total energy (i.e. $e_{\mathcal{E}_{\text {mod }}}^{\text {rel }}(t)$ ) for fixed $\Delta t=10^{-2}$. These results clearly show that the CN-AB-SP scheme is second-order convergent in time. In addition, both the total mass and the original total energy converges at third-order while the modified total energy is very well preserved for all cases, which agrees with Propositions 2.2 and 2.1 .

Example 3.3. In this section, we first apply the CN-AB-SP scheme to simulate the solitonkink interaction in optical fibers and the dynamics of BEC with high-order interaction. To this end, we consider the following cases: 

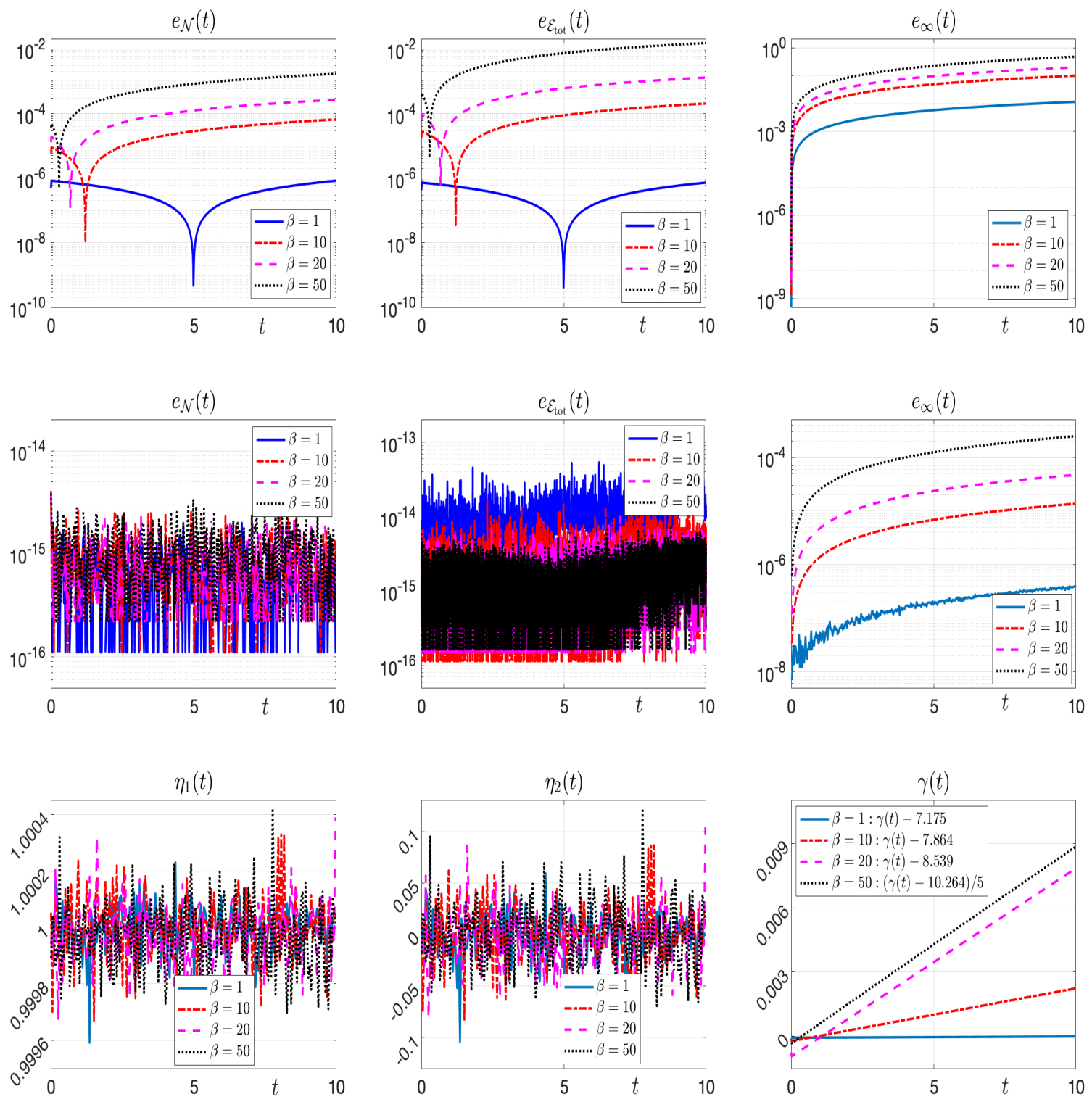

Figure 3.1: Time evolution of the errors on the mass $\left(e_{\mathcal{N}}\right)$, the original total energy $\left(e_{\mathcal{E}_{\text {tot }}}\right)$, the wave function $\left(e_{\infty}\right)$ for the CN-AB-SP (first row) and CN-LF-SP (second row) schemes for Example 3.1 The third row shows the time evolution of the Lagrange multipliers: $\eta_{1}(t)$ and $\eta_{2}(t)$ (for the Lag-GPE formulation) and $\gamma(t)$ (for the standard SAV approach). 

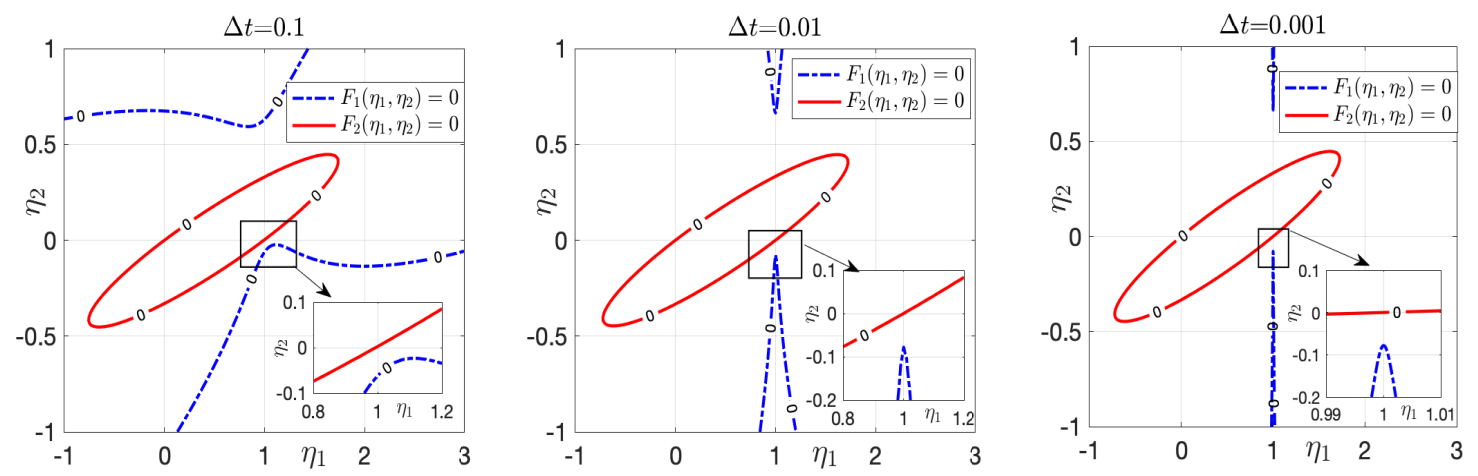

Figure 3.2: The curve of $\mathcal{F}_{1}\left(\eta_{1}, \eta_{2}\right)=0 \& \mathcal{F}_{2}\left(\eta_{1}, \eta_{2}\right)=0$ for the CN-LF-SP scheme with $W=0$ at time $t=2 \Delta t$ in Remark 3.1
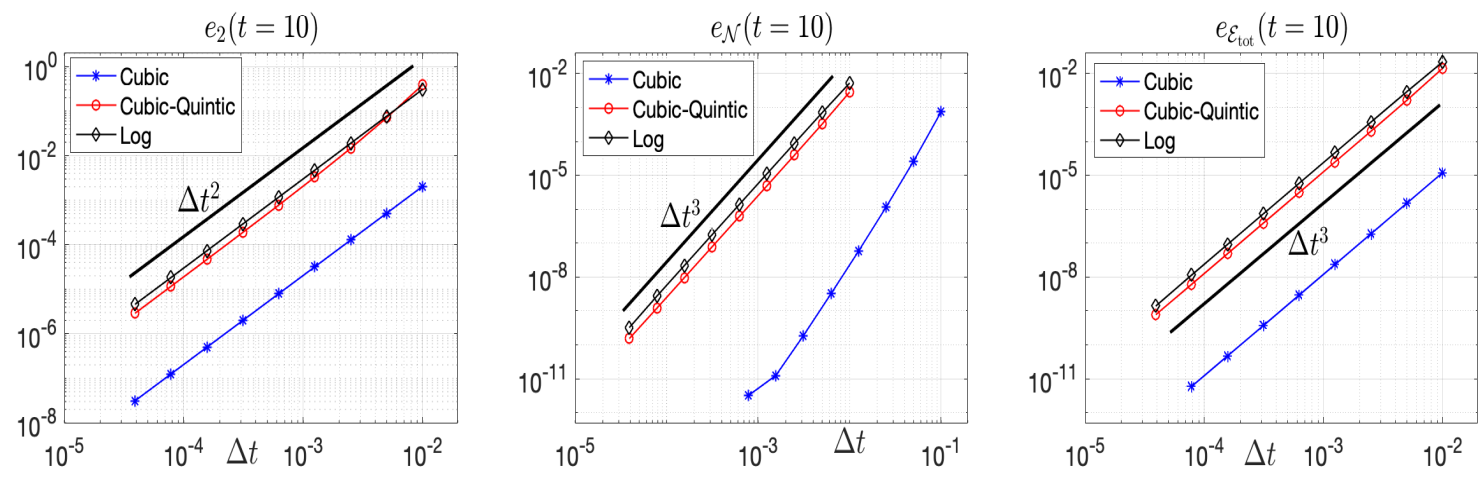

Figure 3.3: The errors $e_{2}(t), e_{\mathcal{N}}(t)$ and $e_{\mathcal{E}_{\text {tot }}}(t)$ vs the time step $\Delta t$ at $t=10$ for Cases 1-3 in Example 3.2
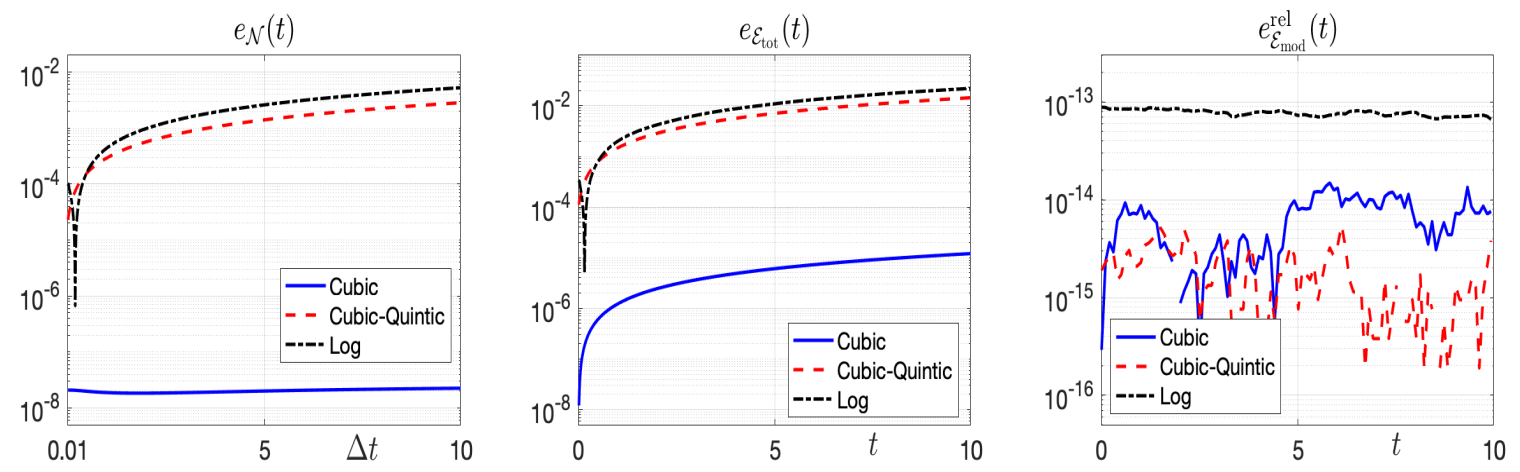

Figure 3.4: Time evolution of the errors on the mass (left), original total energy (middle) and the modified total energy (right) for Cases 1-3 in Example 3.2. The time step is fixed to $\Delta t=10^{-2}$ for all cases. 
- Case 4. To investigate the soliton-kink interaction, we set $V(x)=0$ and $\lambda=0$ and take the cubic-quintic nonlinearity: $f\left(|\psi|^{2}\right)=\alpha_{1}|\psi|^{2}-\alpha_{2}|\psi|^{4}$, with $\alpha_{1}=\alpha_{2}=1$ in (1.1). The initial data is chosen as

$$
\psi_{0}(x)=\phi_{\mathrm{b}}\left(x-x_{\mathrm{b}}^{0}\right)+\phi_{\mathrm{k}}\left(x-x_{\mathrm{k}}^{0}\right)
$$

where the bright soliton $\phi_{\mathrm{b}}(x)$ and kink solution $\phi_{\mathrm{k}}(x)$ reads as

$$
\phi_{\mathrm{b}}(x)=\frac{3 c_{0} e^{i\left(v x / \sqrt{2}+\phi_{0}\right)}}{2 \sqrt{1+c_{0} \cosh \left(3 c_{0} x / \sqrt{2}\right) / 4}}, \quad \phi_{\mathrm{k}}(x)=\frac{\sqrt{3} e^{i \theta_{0}}}{2 \sqrt{1+e^{-\sqrt{3} x / 2}}}
$$

i.e., a bright soliton sit at $x_{\mathrm{b}}^{0}$ while a standing kink locates at the point $x_{\mathrm{k}}^{0}$. Here, we fix $c_{0}=\sqrt{3 / 10}$. Let us remark that, due to the far field behavior of the kink solution [29], and with a similar argument as for the type-I soliton in the cubic NLSE [14, the homogeneous Neumann boundary condition is rather applied at $\partial \mathcal{D}$. Therefore, the Cosine pseudo-spectral method [14] is used for the space discretization instead of the Fourier approximation (2.40). For the computations, the domain, mesh size and time steps are chosen respectively as $\mathcal{D}=[-300,500], h_{x}=1 / 16, \Delta t=10^{-2}$. We fix $x_{0}=-40, v=0.274, \theta_{0}=0$, and consider three different initial phases for the bright soliton

$$
\phi_{0}=-0.4 \pi, \quad \text { or } \quad \phi_{0}=0.2 \pi, \quad \text { or } \quad \phi_{0}=0.9 \pi .
$$

- Case 5. To simulate the dynamics of a BEC with HOI, we set $V(x)=x^{2} / 2$ and take the cubic nonlinearity $f\left(|\psi|^{2}\right)=\beta|\psi|^{2}$ in $(1.1)$. We fix $\beta=1$ and $\lambda=100$. The initial data is chosen as

$$
\psi_{0}(x)=\phi_{\mathrm{g}}\left(x-x_{0}\right),
$$

where $\phi_{\mathrm{g}}(x)$ is the ground state of the NLSE with HOI (1.1) for the same parameters. In the simulation, we take $x_{0}=1$ and $\Delta t=10^{-3}$.

Figure 3.5 depicts the time evolution of the wave function $|\psi|$ as well as the errors on the mass (i.e. $e_{\mathcal{N}}(t)$ ) and on the modified total energy (i.e. $e_{\mathcal{E}_{\text {mod }}}^{\text {rel }}(t)$ ) for Cases 4-5. From this figure we see that: (i) The modified total energy is well conserved. The mass is not conserved but however the errors are small, especially for weak nonlinearities. (ii) The physical phenomena could be correctly captured/computed by the CN-AB-SP scheme, even for the NLSE with strong HOI. (iii) For soliton-kink interaction in cubic-quintic system, radiation waves always occur as long as the soliton meets the kink. In addition, for proper negative impinging phase (i.e. $\phi_{0}<0$ ), the kink acts like a solid wall for bright solitons, which leads to the breakup of the soliton upon it hits the kink. For small positive phases $\phi_{0}$, the impinging bright soliton, upon entering into the region of a higher amplitude of the kink, changes its form instantly into a dark-soliton and then proceeds. For larger positive values of $\phi_{0}$, the bright soliton is reflected back. These phenomena are essentially different from those arising in the cubic NLSE.

\subsection{Two-dimensional case}

In this section, we apply the CN-AB-SP scheme to simulate the dynamics of the 2D GPE. To this end, we fix $\lambda=0$ and consider the cubic nonlinearity, i.e., $f\left(|\psi|^{2}\right)=\beta|\psi|^{2}$ in 

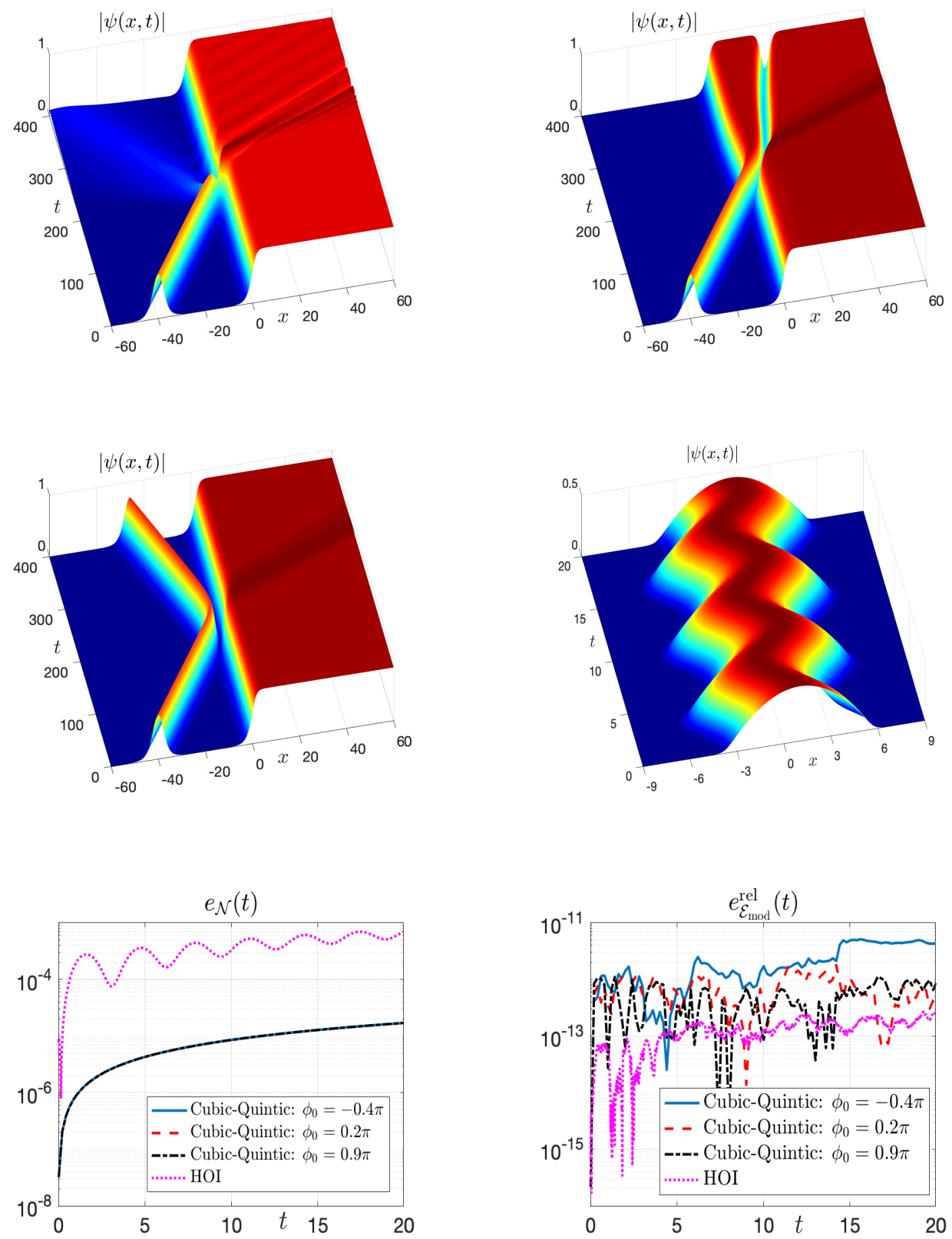

Figure 3.5: Time evolution of the density $|\psi(x, t)|$ for Case 4 with $\phi_{0}=-0.4 \pi$ (top left), $\phi_{0}=0.2 \pi$ (top right), $\phi_{0}=0.9 \pi$ (middle left) and Case 5 (middle right) as well as the errors on mass (i.e. $\left.e_{\mathcal{N}}(t)\right)$ and modified total energy (i.e. $\left.e_{\mathcal{E}_{\text {mod }}}^{\text {rel }}(t)\right)$ for Case 4-5 in Example 3.3 
(1.1). For the dynamics, unless stated otherwise, we take $\mathcal{D}=[-L, L]^{2}, h_{x}=h_{y}=h$. We choose $L=16, h=1 / 8, \Delta t=10^{-4}$ for Cases 6-8 and $L=24, h=1 / 8, \Delta t=5 \times 10^{-5}$ for

Case 9. The "exact solution" when computing the errors in (3.46) is obtained numerically on the same domain with smaller time step $\Delta t=10^{-6}$, and with the same mesh size, i.e. $h_{x}=h_{y}=h=1 / 8$.

Example 3.4. The initial data is chosen as $\psi_{0}(\mathbf{x})=\phi_{\mathrm{g}}\left(\mathbf{x}-\mathbf{x}_{0}\right)$, where $\phi_{\mathrm{g}}(\mathbf{x})$ is the ground state of the GPE with $V(\mathbf{x})=|\mathbf{x}|^{2} / 2$ and following different values of the nonlinear strength $\beta$ and rotation speed $\omega$

- $\quad$ Case 6. $\beta=500, \omega=0 . \quad$ Case 7. $\beta=500, \omega=0.5$.

- $\quad$ Case 8. $\beta=10000, \omega=0.2$. Case 9. $\beta=500, \omega=0.95$.

The computation of the ground state is realized by the preconditioned nonlinear conjugate gradient method with spectral approximation developed in [6]. For the dynamics, we keep the parameters unchanged (except that we change the potential to be $V(\mathbf{x})=|\mathbf{x}|^{2}$ in Case $\mathbf{9})$ and choose $\mathbf{x}_{0}=(3,3)$ for Cases $\mathbf{6}$ and $\mathbf{7}$, and $\mathbf{x}_{0}=(1,1)$ for Case 8.

Figure 3.6 shows the errors $e_{2}(t), e_{\mathcal{N}}(t)$ and $e_{\mathcal{E}_{\text {tot }}}(t)$ vs the time step $\Delta t$ at $t=10$ for Cases 6-8, while Figure 3.7 depicts the time evolution of errors on mass (i.e. $e_{\mathcal{N}}(t)$ ), on the original total energy $\left(\right.$ i.e. $\left.e_{\mathcal{E}_{\text {tot }}}(t)\right)$ and on the modified total energy (i.,e. $\left.e_{\mathcal{E}_{\text {mod }}}^{\text {rel }}(t)\right)$ for Cases 6-9. We can see that the solution is well-approximated at second-order, while the error on the mass and original total energy is numerically of order three. In addition, for a large nonlinearity strength, we observe that the scheme is robust since the time step only needs to be taken slightly smaller for a similar error. This confirms that the choice of the stabilization term $W$ and the constant $E_{c}$ are well-suited. Figure 3.8 shows the trajectory of the center of mass defined by

$$
\mathbf{x}_{c}(t):=\left(x_{c}(t), y_{c}(t)\right)^{T}:=\int_{\mathcal{D}} \mathbf{x}|\psi|^{2} d \mathbf{x}
$$

the time evolution of the components $x_{c}(t)$ and $y_{c}(t)$. Moreover, Figure 3.9 shows the contour plots of the density $|\psi|^{2}$ at different times. For all cases, we see that the path of the center of mass is stable and that the mass grows with time and reaches an error level of $10^{-4}$ even after a relatively long time evolution. The modified total energy is very well-preserved.

\section{Concluding remarks}

In this paper, we propose two linear implicit schemes to solve simulate the dynamics of nonlinear Schödinger equations with various general nonlinearities. One of the schemes is based on the standard SAV approach, while the other one is derived from a SAV formulation via Lagrange multipliers. Coupled with Fourier pseudospectral method for spatial discretization, both schemes are second-order in time and spectrally accurate in space. The two systems are efficient and easy to implement. The standard SAV approach approximates the mass and original total energy at third-order and conserves a modified total energy, while the SAV via Lagrange multiplier approach preserves exactly the mass and original total energy. However, one nonlinear algebraic system needs to be solved at every time step for the 

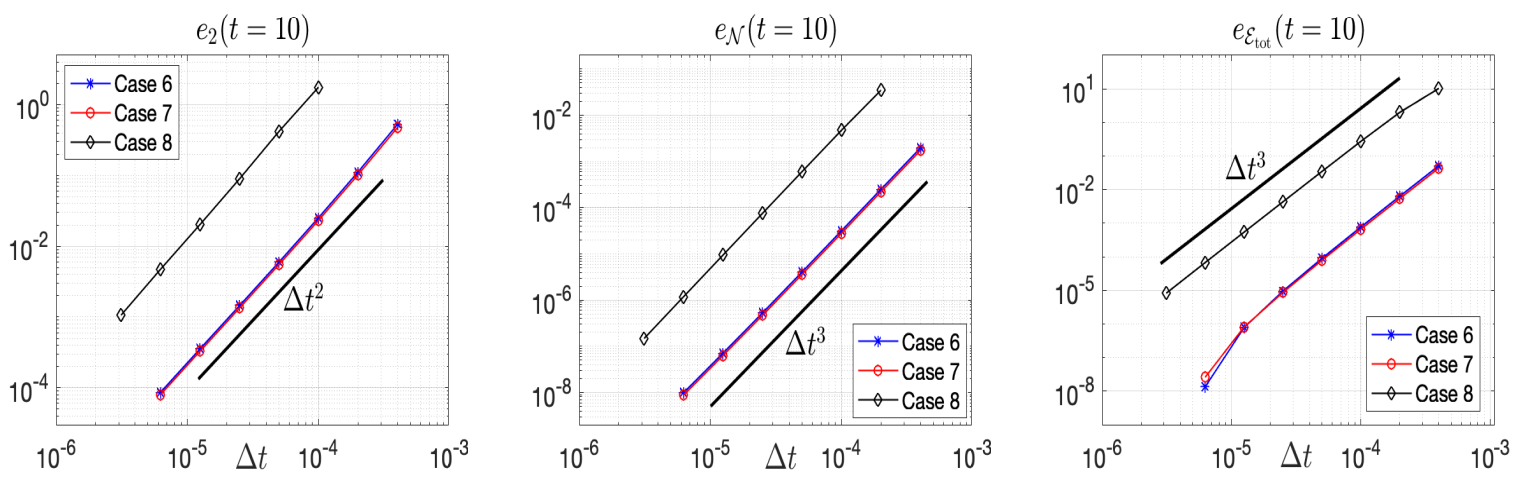

Figure 3.6: The errors $e_{2}(t), e_{\mathcal{N}}(t)$ and $e_{\mathcal{E}_{\text {tot }}}(t)$ vs time step $\Delta t$ at time $t=10$ for Cases 6-8 in Example 3.4
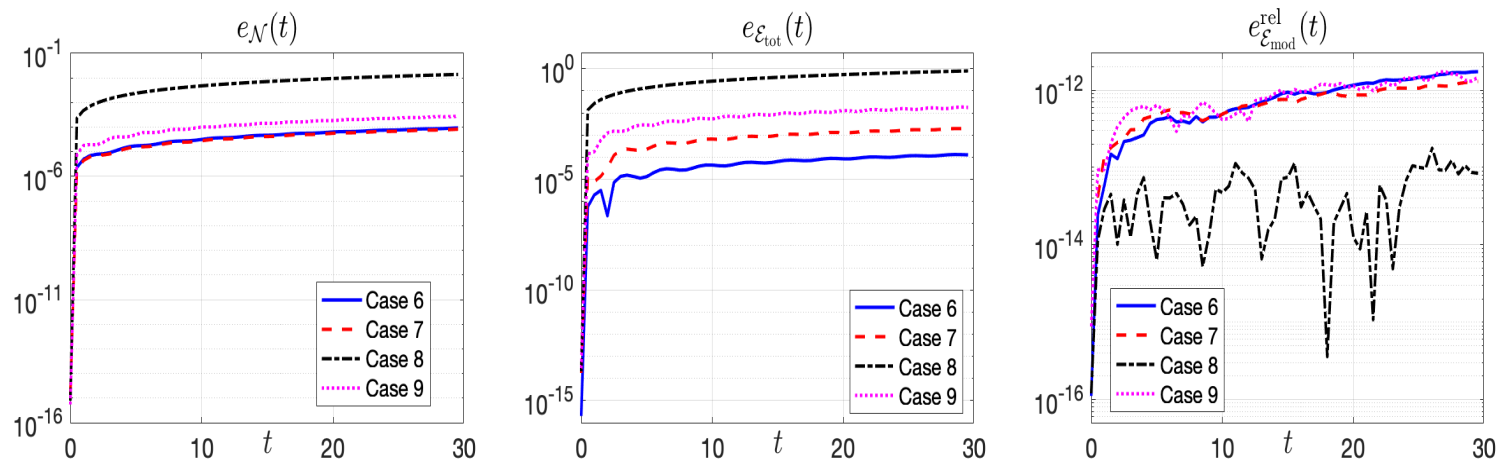

Figure 3.7: Time evolution of the errors on mass (left), original total energy (middle) and modified total energy (right) for Cases 6-9 in Example 3.4 The time step is fixed to $\Delta t=10^{-4}$ for Cases 6-8 and $\Delta t=5 \times 10^{-5}$ for Case 9 .

(a)

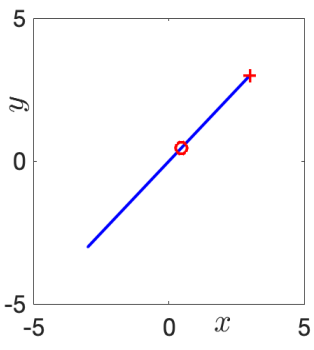

(c)

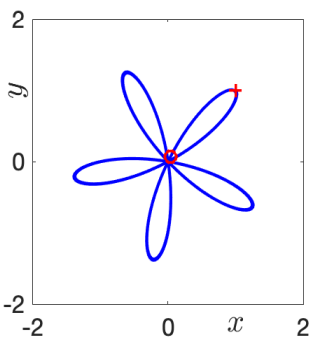

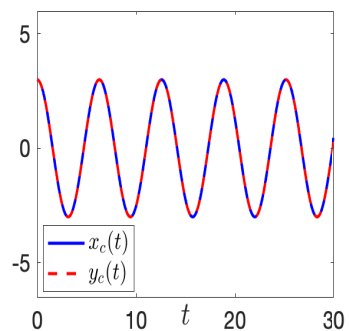

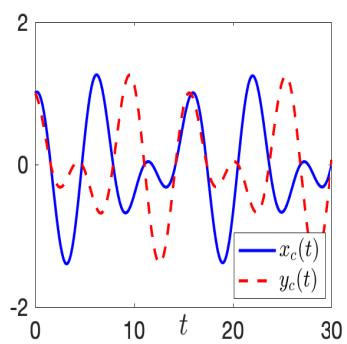

(b)
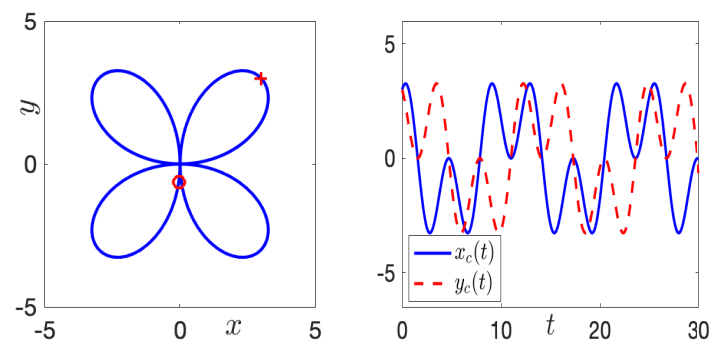

(d)

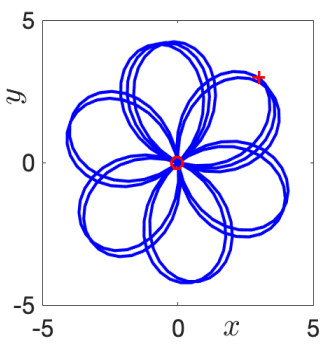

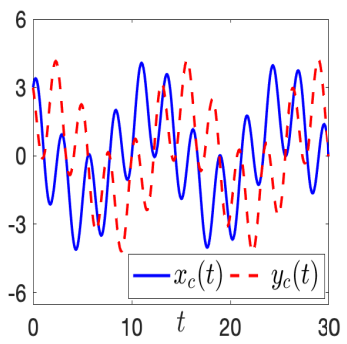

Figure 3.8: Trajectory of the center of mass $\mathbf{x}_{c}$ and time evolution of the $x_{c}(t)$ and $y_{c}(t)$ components in Example 3.4 for Cases 6 - 9 (from (a) to (d)). The time step is fixed to $\Delta t=10^{-4}$ for Cases 6-8 and $\Delta t=5 \times 10^{-5}$ for Case $\mathbf{9}$. 

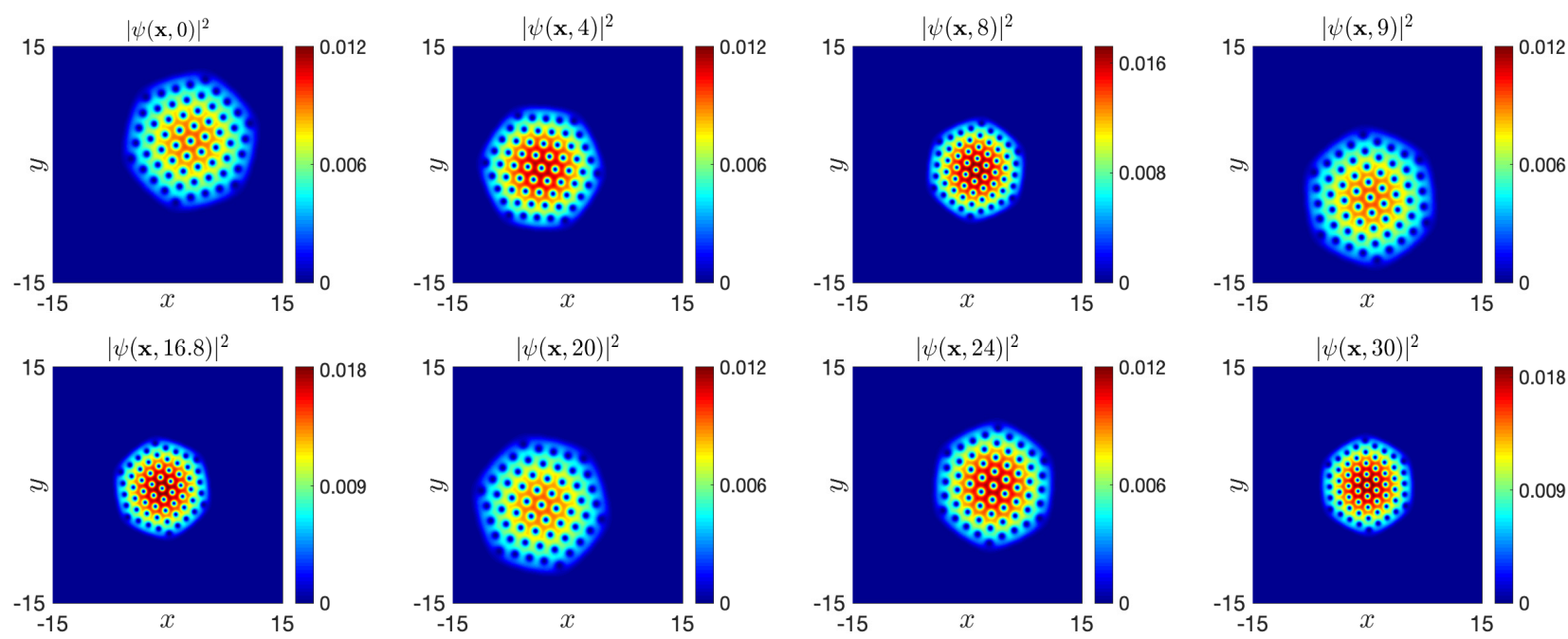

Figure 3.9: Contour plots of the density $\rho=|\psi|^{2}$ at different times for Case 9 in Example 3.4

SAV via Lagrange multiplier approach, the existence of proper real roots (close to its exact solution) is not guaranteed if $\Delta t$ is not sufficiently small. One need then to use a very small time step and/or to adjust wisely the stabilization parameters to proceed with the Lagrange multiplier approach, which could be time consuming. Nevertheless, whenever the Lagrange multiplier approach works, it outperforms the standard SAV approach, for both the errors on the solution, errors on the mass as well as the original total energy. How to more effectively solve/tune the nonlinear algebraic system in the Lagrange multiplier approach is left as a future work. Finally, ample numerical examples illustrate to evidence the accuracy and efficiency of the proposed methods.

\section{Acknowledgements}

This work was partially supported by NSF DMS-2012585 and AFOSR FA9550-20-1-0309 (J. Shen), the Inria through the associated Inria team project BEC2HPC (X. Antoine and Q. Tang), the Fundamental Research Funds for the Central Universities (No. YJ201807) and the National Natural Science Foundation of China (No. 11971335) (Q. Tang).

\section{References}

[1] M. H. Anderson, J. R. Ensher, M. R. Matthewa, C. E. Wieman, E. A. Cornell, Observation of Bose-Einstein condensation in a dilute atomic vapor, Science 269 (1995), pp. 198-201.

[2] X. Antoine, W. Bao and C. Besse, Computational methods for the dynamics of nonlinear Schrödinger and Gross-Pitaevskii equations, (A Feature Article) Comp. Phys. Comm. 184 (12), (2013), pp. 2621-2633.

[3] X. Antoine, C. Besse and V. Rispoli, High-order IMEX-spectral schemes for computing the dynamics of systems of nonlinear Schrödinger/Gross-Pitaevskii equations, J. Comput. Phys., 327, (15), (2016), pp. 252-269. 
[4] X. Antoine, R. Duboscq, Robust and efficient preconditioned Krylov spectral solvers for computing the ground states of fast rotating and strongly interacting Bose-Einstein condensates, J. Comput. Phys. 258 (2014), pp. 509-523.

[5] X. Antoine and R. Duboscq, Modeling and computation of Bose-Einstein Condensates: stationary states, nucleation, dynamics, stochasticity, in Nonlinear Optical and Atomic Systems: at the Interface of Mathematics and Physics, Lecture Notes in Mathematics, 2146, pp. 49-145, Springer, 2015.

[6] X. Antoine, A. Levitt and Q. Tang, Efficient spectral computation of the stationary states of rotating Bose-Einstein condensates by the preconditioned nonlinear conjugate gradient method, J. Comput. Phys. 343, (2017), pp. 92-109.

[7] W. Bao, Y. Cai, Mathematical theory and numerical methods for Bose-Einstein condensation, Kinet. Relat. Mod. 6 (2013), pp. 1-135.

[8] W. Bao, Y. Cai, Optimal error estimates of finite difference methods for the GrossPitaevskii equation with angular momentum rotation, Math. Comput. 82 (2013), pp. 99128.

[9] W. Bao, Q. Du, Y. Zhang, Dynamics of rotating Bose-Einstein condensates and its efficient and accurate numerical computation, SIAM J. Appl. Math. 66 (2006), pp. 758786 .

[10] W. Bao, R. Carles, C. Su and Q. Tang, Error estimates of a regularized finite difference method for the logarithmic Schrödinger equation, SIAM J. Numer. Anal., 57 (2019), pp. 657-680.

[11] W. Bao, D. Jaksch, P. A. Markowich, Numerical solution of the Gross-Pitaevskii equation for Bose-Einstein condensation, J. Comput. Phys. 187 (2003), pp. 318-342.

[12] W. Bao, D. Marahrens, Q. Tang and Y. Zhang, A simple and efficient numerical method for computing the dynamics of rotating Bose-Einstein Condensates via rotating lagrangian coordinates, SIAM J. Sc. Comput. 35(6), (2013), pp. A2671-A2695.

[13] W. Bao, J. Shen, A fourth-order time-splitting Laguerre-Hermite pseudospectral method for Bose-Einstein condensates, SIAM J. Sci. Comput. 26 (2005), pp. 2020-2028.

[14] W. Bao, Q. Tang, Z. Xu, Numerical methods and comparison for computing dark and bright solitons in the nonlinear Schrödinger equation, J. Comput. Phys. 235 (2013), pp. $423-445$.

[15] W. Bao, H. Wang, An efficient and spectrally accurate numerical method for computing dynamics of rotating Bose-Einstein condensates, J. Comput. Phys. 217 (2006), pp. 612626.

[16] C. Besse, A relaxation scheme for the nonlinear Schrödinger equation, SIAM J. Numer. Anal. 42 (2004), pp. 934-952. 
[17] C. Besse, S. Descombes, G. Dujardin and I. Lacroix-Violet, Energy preserving methods for nonlinear Schrödinger equations, IMA Journal of Numerical Analysis, to appear, 2020, (arXiv:1812.04890).

[18] W. Cai, C. Jiang, Y. Wang and Y. Song, Structure-preserving algorithms for the two dimensional sine-Gordon equation with Neumann boundary conditions, J. Comput. Phys., 395 (2019), pp. 166-185.

[19] M. Caliari, Ch. Neuhauser, M. Thalhammer, High-order time-splitting Hermite and Fourier spectral methods for the Gross-Pitaevskii equation, J. Comput. Phys. 228 (2009), 822-C832.

[20] Q. Cheng, C. Liu and J. Shen, A new Lagrange multiplier approach for gradient flows, Comput. Methods Appl. Mech. Engrg., 367 (2020), 13070.

[21] Q. Cheng and J. Shen, Global constraints preserving scalar auxiliary variable schemes for gradient flows, SIAM J. Sci. Comput. 42:A2514- A2536, 2020.

[22] Q. Cheng, J. Shen and X. Yang, Highly efficient and accurate numerical schemes for the epitaxial thin film growth models by using the SAV approach, J. Sci. Comput., 78 (2019), pp. 1467-1487.

[23] A. Collin, P. Massignan and C. J. Pethick, Energy-dependent effective interactions for dilute many-body systems, Phys. Rev. A, 75 (2007), 013615.

[24] M. Delfour, M. Fortin, G. Payre, Finite-difference solutions of a nonlinear Schrödinger equation, J. Comput. Phys. 44 (1981), pp. 277-288.

[25] B. D. Esry and C. H. Greene, Validity of the shape-independent approximation for Bose-Einstein condensates, Phys. Rev. A, 60 (1999), pp. 1451-1462.

[26] X. Feng, B. Li and S. Ma, High-order mass- and energy-conserving SAV-Gauss collocation finite element methods for the nonlinear Schrödinger equation, arXiv:2006.05073.

[27] A. L. Fetter, Rotating trapped Bose-Einstein condensates, Rev. Mod. Phys. 81 (2009), pp. 647-691.

[28] E. P. Gross, Structure of a quantized vortex in boson systems, Nuovo. Cimento. 20 (1961), pp. 454-457.

[29] W. Kim and H. Moon, Dark and bright soliton exchange in a nonlinear dispersive medium, J. Korean Phys. Society, 38 (2001), pp. 558-561.

[30] L. Lin, Z. Yang and S. Dong, Numerical approximation of incompressible Navier-Stokes equations based on an auxiliary energy variable, J. Comput. Phys., 388 (2019), pp. 1-22.

[31] Z. Liu, H. Zhang, X. Qian and S. Song, Mass and energy conservative high-order schemes for the nonlinear Schrödinger equation in one and two dimensions, arXiv:1910.13700v1, October 2019. 
[32] D. Pathria, J. L. Morris, Pseudo-spectral solution of nonlinear Schrödinger equation, J. Comput. Phys. 87 (1990), pp. 108-125.

[33] L. P. Pitaevskii, Vortex lines in an imperfect Bose gas, Soviet Phys. JETP 13 (1961), pp. 451-454.

[34] L. P. Pitaevskii, S. Stringari, Bose-Einstein Condensation (Clarendon Press, Oxford, 2003).

[35] K. I. Pushkarov, D. I. Pushkarov and I. V. Tomov, Self-action of light beams in nonlinear media: soliton solutions, Opt. Quantum Electron., 11(1979), pp. 471-478.

[36] X. Ruan, Y. Cai and W. Bao, Mean-field regime and Thomas-Fermi approximations of trapped Bose-Einstein condensates with higher order interactions in one and two dimensions, J. Phys. B: At. Mol. Opt. Phys., 49 (2016), 125304.

[37] Y. Saad, Iterative Methods for Sparse Linear Systems, (PWS Pub. Co., Boston, 1996).

[38] Y. Saad and M.H. Schultz, GMRES: a generalized minimal residual algorithm for solving nonsymmetric linear systems, SIAM J. Sci. Statist. Comput. 7 (3) (1986), pp. 856-869.

[39] J. Shen and J. Xu, Convergence and error analysis for the scalar auxiliary variable (SAV) schemes to gradient flows. SIAM J. Numer. Anal., 56 (2018), pp. 2895-2912.

[40] J. Shen, J. Xu and J. Yang, The scalar auxiliary variable (SAV) approach for gradient flows, J. Comput. Phys., 352 (2018), pp. 407-417.

[41] J. Shen, J. Xu and J. Yang, A new class of efficient and robust energy stable schemes for gradient flows. SIAM Review, 61 (2019), pp. 474-506.

[42] J. Shen and Q. Zhuang, Efficient SAV approach for imaginary time gradient flows with applications to one- and multi-component Bose-Einstein Condensates. J. Comput. Phys. 396 (2019), pp. 72-88.

[43] M. Thalhammer, High-order exponential operator splitting methods for time-dependent Schrödinger equations, SIAM J. Numer. Anal. 46 (2008), pp. 2022-2038.

[44] M. Thalhammer, Convergence analysis of high-order time-splitting pseudo-spectral methods for nonlinear Schrödinger equations, SIAM J. Numer. Anal. 50 (2012), pp. 3231-3258.

[45] H. Wang, A time-splitting spectral method for coupled Gross-Pitaevskii equations with applications to rotating Bose-Einstein condensates, J. Comput. Appl. Math. 205 (2007), pp. 88-104.

[46] J. A. C. Weideman, B. M. Herbst, Split-step methods for the solution of the nonlinear Schrödinger equation, SIAM J. Numer. Anal. 23 (1986), pp. 485-507. 